\title{
Jutta Müller-Tamm
}

\section{Das geteilte Berlin als Katalysator der Internationalisierung des Literaturbetriebs}

\section{I.}

Nach dem Mauerbau war Berlin eine doppelt isolierte Metropole. Aus ostdeutscher Perspektive handelte es sich - der offiziellen Wendung zufolge - um die Befestigung der Staatsgrenze durch einen ,antifaschistischen Schutzwall'. Für Kulturschaffende im Osten, die wie Stefan Hermlin den Mauerbau als Akt der Verteidigung rechtfertigten, ${ }^{1}$ mochte sich die Schließung der Grenze zunächst mit der Hoffnung auf größere Freiheit im eigenen Land verbinden, weil man jetzt ,unter sich“ war und ,alles sagen' konnte. De facto bedeutete sie aber nicht nur die Einsperrung der eigenen Bürger, sondern auch die Selbstisolation der DDR und nicht zuletzt eine kulturelle Abschottung. Eingemauert waren wiederum auch und erst recht die drei westlichen Sektoren Berlins, umschlossen durch die DDR. Für den Westteil der Stadt drohten in der Folge Abwanderung, schwindende Wirtschaftskraft, kulturelle Marginalisierung: die Bedeutungslosigkeit und Provinzialität einer insularen Stadt.

Auf beiden Seiten der Grenze brachte diese Situation ein gesteigertes Bemühen um kulturelle Geltung hervor. Ein wichtiges Mittel in diesem Bemühen war die Förderung internationaler Kontakte. Aus dieser Beobachtung ergibt sich die grundlegende These des vorliegenden Bandes, der zufolge die Mauer, so paradox dies klingen mag, als Katalysator für die Internationalisierung des Literatur- und Kulturbetriebs wirkte. Sie war ein entscheidender Akteur in jener Entwicklungsphase, in der Berlin mit Blick auf die transnationale Ausweitung des Literaturbetriebs eine Vorreiterfunktion übernahm. Zwar gab es auch schon in den 1950er Jahren internationale Aktivitäten auf Berliner Boden: So wurden beispielsweise 1951 im Westteil der Stadt die Berliner Festwochen gegründet, die jährlich stattfindenden internationalen Festspiele vor allem für Musik und Theater, auf die 1957 - sechs Jahre später - die Parallelgründung der Berliner Festtage im Ostteil der Stadt folgte. Bereits hier zeigt sich eine Konkurrenz um Weltgeltung und das Streben, sich gegen den anderen Teil der Stadt auf dem internationalen Parkett zu behaupten. ${ }^{2}$ Dennoch lässt sich feststellen, dass 1961 auf beiden Seiten der Mauer eine intensivierte Phase der kulturpolitischen „Aufrüstung“ begann, in deren Zentrum die gezielte Förderung internationaler literarischer und künstlerischer Kontakte stand.

1 Stephan Hermlin: Offener Brief an Wolfdietrich Schnurre und Günter Grass, 17. August 1961, in: Hans Werner Richter (Hrsg.): Die Mauer oder Der 13. August, Reinbek bei Hamburg 1961, 66-68. 2 Vgl. Kerstin Decker: Kunst ist Waffe? Die Berliner Festwochen als Spezialfall der Ostpolitik, in: Henrik Adler, Joachim Sartorius (Hrsg.): Das Buch der Berliner Festspiele, Berlin 2011, 127-139. 
Die These vom geteilten Berlin als Katalysator der Internationalisierung wird aber nur dann verständlich, wenn man verfolgt, auf welche Art und Weise die Bemühungen einer Internationalisierung in Ost und West miteinander zusammenhängen und wie sie sich wechselseitig aufeinander beziehen. Auch wenn bei diesen Aktivitäten in Westund Ostberlin divergierende Absichten verfolgt, unterschiedliche Konzepte von Weltliteratur verhandelt und konkurrierende Gemeinschaften gestiftet wurden, handelt es sich doch um miteinander verknüpfte, ja oftmals voneinander abhängige Prozesse.

Dieser Grundgedanke entspricht dem Ansatz der neueren zeitgeschichtlichen und literaturhistorischen Forschung: Diese strebt auf ihren jeweiligen Feldern eine integrierte Darstellung der deutsch-deutschen Nachkriegsgeschichte an, insofern sie von Trennung und Divergenz, aber auch von Parallelität und Verflechtung im Verhältnis beider Systeme ausgeht. So hat Petra Weber jüngst in ihrer großangelegten Gesamtdarstellung der Jahre 1945 bis 1989/90 den Anspruch formuliert, „die deutschdeutsche Geschichte als Parallel-, Kontrast-, Vergleichs-, Perzeptions- und Beziehungsgeschichte zu erzählen. “3 Für die deutsch-deutsche Literaturgeschichte wurde ein entsprechender Ansatz auf je unterschiedliche Weise von Helmut Peitsch, Heribert Tommek u. a. stark gemacht. ${ }^{4}$ Auch die konkreten literarischen Beziehungen zwischen Ost und West sind intensiv beforscht worden, und zwar die offiziellen wie die unterdrückten und inoffiziellen, so etwa, um nur ein Beispiel zu nennen, in dem von Roland Berbig herausgegebenen Band Stille Post. ${ }^{5}$

Der Ansatz einer Beziehungs- und Verflechtungsgeschichte liegt auch den folgenden Ausführungen zugrunde. Im Zentrum dieses Aufsatzes wie des Bandes insgesamt stehen jedoch nicht die beiden deutschen Literaturen und ihr Verhältnis zueinander; vielmehr interessieren hier die nicht-deutschen Autor:innen und die nicht-deutschen Literaturen in Berlin. Es geht um die wechselseitige Aktivierung und Motivierung der internationalen Bemühungen in Ost und West, um das Widerspiel in der Außenorientierung: wie man, gewissermaßen Rücken an Rücken und in permanenter Tuchfühlung, um internationale Kontakte und internationale Geltung konkurrierte. Diese merkwürdige, bisweilen paradox erscheinende Geschichte der aufeinander bezogenen Internationalisierungsbemühungen auf literarischem Gebiet - des Ineinanders von Abgrenzung, Entsprechung und Verflechtung in diesen Bestrebungen - ist noch nicht geschrieben worden, und, soweit sie sich auf Berliner Boden bewegt, ist sie der Gegenstand des vorliegenden Aufsatzes und des gesamten Bandes.

3 Petra Weber: Getrennt und doch vereint. Deutsch-deutsche Geschichte 1945-1989/90, Berlin 2020, 15. 4 Helmut Peitsch: Nachkriegsliteratur 1945-1989. Göttingen 2009; Heribert Tommek: Die Formation der Gegenwartsliteratur. Deutsche Literaturgeschichte im Lichte von Pierre Bourdieus Theorie des literarischen Feldes, in: IASL 40.1 (2015), 110-143.

5 Roland Berbig (Hrsg.): Stille Post. Inoffizielle Schriftstellerkontakte zwischen Ost und West, Berlin 2005. Vgl. auch Julia Frohn: Literaturaustausch im geteilten Deutschland 1945-1972, Berlin 2014. 


\section{II.}

Der Mauerbau bedeutete eine Zäsur auch für die Situation der Literatur und ihrer Akteure in Berlin; und er hatte Konsequenzen für die Kulturpolitik zunächst vor allem im Westen. Auf die drastische ,Regionalisierung ' Westberlins durch den Mauerbau reagierte man naheliegenderweise durch Verstärkung der Außenkontakte.

Eine erste Unternehmung in dieser Richtung war die Berlin Stiftung für Literatur und Sprache, die der Kulturkreis im Bundesverband der Deutschen Industrie im November 1961 ins Leben rief. Deren Ziel bestand darin - so kolportierte es die westdeutsche Presse -, „,im heutigen Berlin führende Europäer auf einem literarischen Forum zu Gegenwartsproblemen Stellung nehmen zu lassen. Dichter, Philosophen und Kritiker von internationalem Rang sollen sich auf Einladung des Verbandes jeweils längere Zeit in Berlin aufhalten, Kontakt zur Berliner Bevölkerung finden und die geistigen Kräfte kennenlernen, von denen der Widerstandswille Berlins getragen wird. Diese Einladungen werden zum allgemeinen Verständnis des Ost-West-Problems beitragen und gleichzeitig zur wechselseitigen Beschäftigung mit der europäischen Kultur anregen.“6

In der Tat belebte sich die literarische Szene in den folgenden Jahren. Hans Werner Richter, der Begründer und Promotor der Gruppe 47, schrieb in einem Brief vom Dezember 1961, er habe nach einem Berlinbesuch die Absicht, sich in Berlin eine Wohnung $\mathrm{zu}$ besorgen und zeitweise dort zu leben. Vier Monate nach dem Mauerbau bejahte er ausdrücklich die Frage, ob Berlin immer noch die Metropole Deutschlands sei:

\footnotetext{
Berlin hat keine Nachfolge gefunden. Nur Berlin und seine kosmopolitisch denkende Bevölkerung besitzt die Voraussetzungen als Hauptstadt Deutschlands. Es wird niemals einen Ersatz dafür geben.

[...] „Was kann man tun?“ Mit anderen Worten, was würde eine auch nur halbwegs nationalbewußte Bevölkerung tun, um seine Hauptstadt vor dem langsamen Erstickungstod zu retten? Die Antworten sind mannigfaltig, aber fast alle kulturpolitischer Art - also alle großen Zeitungen müßten nach Berlin verlegt werden, alle Intellektuellen nach Berlin ziehen, kulturpolitische Zeitschriften müßten in Berlin erscheinen usw. Was mir als Vorschlag generell vorschwebt ist: Berlin zu einem deutschen kulturpolitischen Zentrum von internationalem Rang zu machen. Dies ist möglich, wenn man es ernsthaft will. Ein Beispiel ist Höllerer, der schon sehr viel in dieser Hinsicht unternimmt. Aber man darf dies nicht untergeordneten Beamten überlassen. Dann wird es nie etwas. ${ }^{7}$
}

\footnotetext{
6 Paul Florian: ,Olympische Spiele der Literatur. Berlin: Treffpunkt internationaler Dichter-Prominenz, in: Pforzheimer Zeitung (27.11.1961).

7 „Hans Werner Richter an Georg Ramsegger“, in: Die Welt (04.12.1961), zit. nach: Jürgen Schutte, Elisabeth Unger, Irmtraud Gemballa (Hrsg.): Dichter und Richter. Die Gruppe 47 und die deutsche Nachkriegsliteratur (Katalog der Ausstellung der Akademie der Künste vom 28. Oktober bis 7. Dezember 1988), Berlin 1988, 277.
} 
Nicht allein Hans Werner Richter und nicht nur Walter Höllerer, von dem noch die Rede sein wird, sondern auch einige Beamte kamen auf die Idee, das eingeschlossene Berlin zu einem kulturpolitischen Zentrum von internationalem Rang zu machen. Die offizielle politische Strategie bestand darin, Westberlin als Kulturstandort auszurufen: Nach dem Mauerbau wurde - so die wiederholt vorgetragene und in der Presse kolportierte Formulierung - „das Kulturzentrum Berlin“ proklamiert. $^{8}$

Ausschlaggebend für den Erfolg dieser Kulturoffensive war der Umstand, dass man US-amerikanische Geldgeber fand, denen es im Sinne einer stärkeren Westbindung zupasskam, den Berlinern unter die Arme zu greifen. Die internationalen Aktivitäten der folgenden Jahre wurden in entscheidendem Maße durch die Ford Foundation gefördert, die seinerzeit finanzkräftigste philanthropische Stiftung der Welt. Sie war 1936 vom Autohersteller Ford gegründet worden mit dem erklärten Ziel, Demokratie zu verbreiten, Armut zu reduzieren und die internationale Verständigung zu fördern. In Zeiten des Kalten Krieges verbarg sich dahinter aber durchaus eine dezidierte Ideologie, eine deutlich vertretene antikommunistische Linie. Diese politische Agenda wurde in den 1950er Jahren sehr entschieden, in den 1960er Jahren weniger aggressiv verfolgt, man wollte nun auch die kritischen Intellektuellen einbeziehen. Tatsächlich hatte allerdings der US-amerikanische Geheimdienst CIA bei der Ford Foundation die Hände im Spiel, dies kam 1967/68 ans Licht, ein veritabler Skandal, der dazu führte, dass der von der CIA und der Ford Foundation finanzierte Congress for Cultural Freedom, eine 1950 gegründete antikommunistische Organisation, seine Arbeit beenden musste. ${ }^{9}$ Noch im Berlin der frühen 1960er Jahre jedoch trat die Ford Foundation als diejenige Instanz in Erscheinung, die ein zukunftsweisendes Kulturprogramm ermöglichen sollte. Der Senator für Wissenschaft und Kunst veröffentlichte in Form einer Pressemitteilung nachstehende Erfolgsmeldung:

Im Zuge der nach dem 13. August 1961 eingeleiteten Bemühungen, Berlin in verstärktem Maße zu einem Zentrum der Bildung, der Wissenschaft und der Kunst auszubauen, erklärte sich 1962 die Ford Foundation bereit, für einen Zeitraum von etwa drei Jahren insgesamt etwa 8 Millionen Mark für ein besonderes Programm zur Verfügung zu stellen. ${ }^{10}$

Der Jahresbericht der Ford Foundation von 1963 vermerkt, was mit dieser stattlichen Summe für die Berliner Kultur initiiert wurde: ein Internationales Institut

8 Vgl. „Berlin wird zum Kulturzentrum“, in: Berliner Morgenpost (09.01.1963); „Denn rauhes Klima prägt“, in: Berliner Morgenpost (18./19.11.1964); Helmut Böttiger: Elefantenrunden. Walter Höllerer und die Erfindung des Literaturbetriebs, Berlin 2005, 174.

9 Zu den Aktivitäten der Ford Foundation vgl. Francis Stonor Saunders: Wer die Zeche zahlt ... Der CIA und die Kultur im Kalten Krieg, Berlin 1999; Volker Berghahn: Transatlantische Kulturkriege. Shepard Stone, die Ford-Stiftung und der europäische Antiamerikanismus, Stuttgart 2004.

10 Aus einer Pressemitteilung des Senators für Wissenschaft und Kunst 1963, zit. nach: Stefanie Endlich, Rainer Höynck (Hrsg.): Blickwechsel. 25 Jahre Berliner Künstlerprogramm, Berlin 1988, 26. 
für Vergleichende Musikstudien, das nachmals und bis heute nach John F. Kennedy benannte Institut für Nordamerikastudien an der Freien Universität, ein Artists in Residence-Programm, das ab 1966 als Berliner Künstlerprogramm vom Deutschen Akademischen Auslandsdienst übernommen wurde, sowie das Literarische Colloquium Berlin. ${ }^{11}$

\section{III.}

Peter Nestler, erster Leiter des Berliner Künstlerprogramms nach dem in der Initialphase verantwortlichen russisch-amerikanischen Komponisten und Schriftsteller Nicolas Nabokov, betonte den herausragenden Stellenwert dieser Unternehmung innerhalb der Aktivitäten der Ford Foundation, wenn er von dem „besonders spektakulären Auftakt“ des Künstlerprogramms sprach und darauf hinwies, dass es „den Initiatoren [...] innerhalb ganz kurzer Zeit [gelang], Schriftsteller und Komponisten von Weltrang nach Berlin einzuladen. "12 Dabei beruhte die Einladungspolitik zunächst vor allem auf informellen Verbindungen zu renommierten westlichen Künstlern man folgte dem „mehr patriarchalischen Einladungsprinzip des ,bring your famous friends ““. ${ }^{13}$ Die Internationalisierungsbemühungen richteten sich in dieser Phase entschieden auf große Namen und den repräsentativen Kulturbetrieb. Einen Coup landete die Ford Foundation, indem sie gleich zu Beginn des Programms Witold Gombrowicz nach Berlin einladen konnte. Gombrowicz, einer der wichtigsten polnischen Autoren des 20. Jahrhunderts, hatte fast 25 Jahre im argentinischen Exil verbracht und kehrte erstmalig als Gast des Künstlerprogramms wieder nach Europa zurück. Nach seinem Berliner Jahr blieb er in Europa und nahm seinen Wohnsitz in Frankreich. Mit ihm waren - in der Sparte Literatur - Ingeborg Bachmann (Österreich), Piers Paul Read (Großbritannien), Joachim Prenzlow und Klaus Roehler (Westdeutschland), im darauffolgenden Jahr 1964 W. H. Auden und Eric Bentley (USA), Elazar Koppel Benyoetz (Israel), Michel Butor (Frankreich) und Hans-Dietrich Sander (Westdeutschland)

11 „A three-year program to expand the artistic, educational, and cultural resources of Berlin was inaugurated with a \$2 million appropriation. [...] Initial grants included $\$ 300,000$ to the Free University of Berlin for American Studies and \$350,000 for an International Institute for Comparative Music Studies, where musical traditions of Asia and Africa as well as the West will be studied. A total of $\$ 590,000$ was committed to enable artists, writers, educators, scientists, and composers to visit and work in Berlin for extensive periods. Included was support for a Literary Colloquium, part of whose program will be concerned with the increased use of literature on radio, television, and film. “ The Ford Foundation Annual Report 1963, 49. Online abgerufen am 27. März 2021 auf der Website der Ford Foundation unter https://www.fordfoundation.org/media/2433/1963-annual-report.pdf.

12 Peter Nestler, Das Berliner Künstlerprogramm. Vorläufe und Anfänge, in: Blickwechsel (Anm. 10), 59-62, hier: 59.

13 Ebd., 61. 
eingeladen. Gemeinsam mit den Gästen aus den Bereichen Musik und Bildende Kunst sollten sie die Kulturszene der Stadt mit den gegenwärtigen Strömungen und Maßstäben der westlichen, internationalen Moderne bekannt machen und so - in Form einer „kulturellen Nothilfe“, ${ }^{14}$ wie es die Berliner Schriftstellerin Ingeborg Drewitz formulierte - die Standortprobleme ausgleichen sowie den Anschluss an das führende westliche Kulturleben gewährleisten.

Bis in die 1970er Jahre bestand allerdings ein Problem darin, dass das Künstlerprogramm zwar renommierte Persönlichkeiten nach Berlin holte, die Integration in die ansässige Kulturszene aber nicht wirklich gelang. Zum einen fühlten sich Westberliner Künstler:innen gegenüber der üppig finanzierten internationalen Prominenz zurückgesetzt, zum anderen aber litt die Verständigung an den Sprachbarrieren. Klaus Roehler, Mitglied der Gruppe 47, gehörte zu den wenigen deutschen bzw. deutschsprachigen Gästen in der Frühzeit des Programms. Er schildert das Beisammensein der „,in Britz, in Reinickendorf oder Spandau“ untergebrachten Stipendiaten, die sich „gelegentlich auf einer sogenannten Cocktailparty, zu der der besorgte Vertreter der Ford Foundation in Berlin bittet“, treffen:

Es fällt ihm [dem Stipendiaten, d. i. Roehler selbst] schwer, sich mit allen Stipendiaten zu verständigen. Der Schriftsteller aus Polen etwa, der in Südamerika lebt, spricht Polnisch, spricht Spanisch und dazu ein hartes, polnisch-spanisches Französisch, der Komponist aus Japan spricht fließend Japanisch, darüber wundert sich freilich niemand, und kann auf Englisch sagen „How do you do“ und „Good bye“, der Maler aus Italien spricht Italienisch, das ist sein gutes Recht, und die Frau des Malers übersetzt auf Wunsch ins Englische oder Deutsche, dennoch verlaufen die Gespräche mit dem Maler fast so eintönig wie Gespräche mit dem Schriftsteller aus Polen, der in Südamerika lebt, mit dem Komponisten aus Japan, denn während die Frau des Malers übersetzt, wird der Maler, weil er nicht mithören kann, was seine Frau sagt, unruhig, und deshalb gibt sie seine gewöhnlich lange Rede gekürzt weiter, faßt sich auch der Antwortende kurz, übersetzt darauf die Frau dem Mann die kurze Antwort gekürzt. So scheitern manche gerade erst hoffnungsvoll begonnenen Gespräche an der Vielzahl der bekannten Sprachen, obwohl der Stipendiat sich gerade mit dem italienischen Maler, dem japanischen Komponisten gerne unterhalten hätte; er soll ja aber, und vielleicht ist das von seinen Mäzenen vorausbedacht worden, nicht so viel reden in Berlin, er soll malen, komponieren, schreiben, muß es freilich nicht tun, doch es wird gern gesehen ... ${ }^{15}$

Man sieht, wie hier Anspruch und Möglichkeit von Internationalität stark auseinanderklaffen; weder war die Zeit der allgemeinen Verkehrssprache Englisch angebrochen, noch hatten sich in Berlin - wie heutzutage - mehrsprachige künstlerische Communities oder Szenen herausgebildet. Die Vision einer „Ford Foundation

14 Ingeborg Drewitz: Kulturelle Nothilfe für Berlin? oder Das Berliner Künstlerprogramm (pro contra - pro), in: Deutscher Akademischer Austauschdienst (Hrsg.): 10 Jahre Berliner Künstlerprogramm, Berlin 1975, 39-40.

15 Klaus Roehler: Beitrag für das RIAS-Abendstudio (1965), zit. nach: Blickwechsel (Anm. 10), 26. 
Familie“, ${ }^{16}$ wie sie der Berliner Vertreter der Stiftung beschwor, ließ sich nicht wirklich umsetzen; und das Prinzip, „im internationalen Repräsentationswert der eingeladenen Gäste eine Qualität für den Austausch zu sehen, darauf vertrauend, daß mit der Anwesenheit von Internationalen die ,Internationale‘ selbst ihren Einzug feiere“, ${ }^{17}$ hatte sich nicht bewährt. Nach anderthalb von drei Jahren Finanzierung durch die Ford Foundation fiel die Bilanz für das Artists in Residence-Programm in der Presse eher negativ aus: „Es fragt sich jedoch, ob es - nach den Erfahrungen, die man gemacht hat - überhaupt sinnvoll ist, ausländische Künstler mit Geld und guten Worten für ein ganzes Jahr nach Berlin zu locken. Eine Handvoll prominenter Namen - gewissermaßen als ,Glanzlichter' aufgesetzt - macht aus einer Stadt noch kein Kulturzentrum.“18

\section{IV.}

Wendet man sich den spezifisch auf Literatur bezogenen Aktivitäten in der Internationalisierungsgeschichte der Berliner Kulturszene zu, kommt einer der wichtigsten Akteure in diesem Feld der frühen 1960er Jahre ins Spiel: der bereits erwähnte Walter Höllerer, Literaturwissenschaftler, Zeitschriftengründer, Schriftsteller, Lyriker, Mitglied der Gruppe 47. Seit 1959 hatte er eine Professur an der Technischen Universität Berlin inne, wo er das Institut für Sprache im technischen Zeitalter gründete. Vor allem aber war er ein begnadeter Kulturvermittler und -manager, die zentrale Figur am Schnittpunkt von Universität, Kultur, Wirtschaft und Politik. Höllerer hatte entscheidenden Anteil an der Veränderung, Ausgestaltung und Internationalisierung der literarischen Verhältnisse in Westberlin. Man hat mit Blick auf Höllerer auch von der „Erfindung des Literaturbetriebs“19 gesprochen, um damit seine Scharnierrolle in den Wandlungsprozessen des literarischen Feldes zu beschreiben, seine Kontakte zu Wirtschaft und Politik, seine Managerqualitäten und seine Beherrschung der neueren medialen Formate, also insbesondere Radio und Fernsehen. ${ }^{20}$

16 Brief von Karl Haas, Ford Foundation, an Walter Höllerer vom 8. Oktober 1963. Nachlass Walter Höllerer, Literaturarchiv Sulzbach-Rosenberg, Signatur 03WH/DJ/A/4, 32.

17 Lothar Romain: Kulturpolitische Aspekte des Berliner Künstlerprogramms, in: 10 Jahre Berliner Künstlerprogramm (Anm, 14), Berlin 1975, 17-21, hier: 20.

18 Cornelia Jacobsen: „Halbzeit bei der Ford-Stiftung. Warum viele der eingeladenen Künstler in Berlin unzufrieden sind“, in: Die Zeit Nr. 40 (02.10.1964).

19 Helmut Böttiger: Elefantenrunden. Walter Höllerer und die Erfindung des Literaturbetriebs, Berlin 2005.

20 Vgl. hierzu vor allem Michael Peter Hehl: Berliner Netzwerke. Walter Höllerer, die Gruppe 47 und die Gründung des Literarischen Colloquiums Berlin, in: Achim Geisenhanslüke, Michael Peter Hehl (Hrsg.): Poetik im technischen Zeitalter. Walter Höllerer und die Entstehung des modernen Literaturbetriebs, Bielefeld 2013, 155-189. 
Ein wichtiges Signal setzte Höllerer mit seiner Lesereihe ,Literatur im technischen Zeitalter‘, die im Winter 1961/62 in internationaler Besetzung stattfand. Autor:innen des Nouveau Roman waren eingeladen, der Skandalautor Henry Miller und mit Dos Passos der wichtigste Vertreter des amerikanischen Großstadtromans: Die aktuelle internationale Moderne in unterschiedlichen Stimmen und Sprachen kam in das große Auditorium der Kongresshalle. Es war das Berliner Großereignis des Winters, die Kongresshalle war immer überfüllt, der Sender Freies Berlin nahm die Veranstaltungen auf und strahlte sie im Abendprogramm aus. In seiner Einführung wies Höllerer eigens auf die technischen Gerätschaften hin, denen die Vortragenden, aber auch die $\mathrm{Zu}$ schauer durch die Aufnahmen ausgesetzt wären:

Die Störungen, die die Apparaturen von Rundfunk und Fernsehen notwendigerweise für Sie, die Anwesenden im Saal, mit sich bringt [sic!], werden Sie sicherlich mit Verständnis hinnehmen; wenn Sie bedenken, daß diese Apparatur es ermöglicht, denjenigen unserer bisherigen Studenten und denjenigen Mitbürgern, die wir gerne hier hätten, die gerne kämen, die aber gehindert werden zu kommen, diese Lesereihe zu übermitteln. ${ }^{21}$

Wenige Monate nach dem Mauerbau rief Höllerer also die mediale Möglichkeit ins Bewusstsein, die internationale Lesereihe auch nach Ostberlin auszustrahlen. Dabei gehörte es unabhängig von der deutsch-deutschen Situation und der Übertragung von Fernseh- und Radiosendungen in den Osten zu Höllerers Programm, Literatur und Massenmedien einander anzunähern: Im technischen Zeitalter gelte es, „neuartige Formen der Literatur“ auszuprobieren, „die Einfluß auf die Massenmedien nehmen: Literatur, die dem Fernsehen, dem Film, den Zeitungen Auftrieb gibt“. ${ }^{22}$ Dennoch blieb das Motiv, mit Radio und Fernsehen die Mauer überwinden zu können, eine Konstante: „Television and radio are the only methods of breaking down the Wall“, ${ }^{23}$ wie Höllerer in einem Interview für die Zeitschrift The Atlantic Monthly formulierte.

Die Vertiefung dieser Aktivitäten und ihre Institutionalisierung in Form eines permanenten Literarischen Colloquiums wurden wiederum durch die Ford Foundation ermöglicht. Höllerer kam über einen Bekannten in Kontakt mit Shepard Stone, dem Leiter des internationalen Programms der Stiftung. Dieser Mittelsmann war Walter Hasenclever, der Schwager von Shepard Stone, der dann auch der erste Geschäftsführer des Literarischen Colloquiums werden sollte. In einem Brief an Stone vom 27. Juli 1962 entwarf Höllerer das Programm einer literarischen Institution, zu deren Finanzierung er die Ford Foundation bewegen wollte:

21 Einführung zur Auftaktveranstaltung, Internationale Lesereihe Literatur im technischen Zeitalter, (13.11.1961). Nachlass Walter Höllerer, Literaturarchiv Sulzbach-Rosenberg, Signatur 03WH/CA/3,4.

22 Walter Höllerer, Brief an Shepard Stone vom 27. Juli 1962. Nachlass Walter Höllerer, Literaturarchiv Sulzbach-Rosenberg, Signatur 03WH/DJ/A/4,7.

23 Walter Höllerer, Günter Grass, Walter Hasenclever: „Writers in Berlin. A Three-way Discussion“, in: The Atlantic Monthly (Dezember 1963), 110-113, hier: 113. 


\begin{abstract}
Man erwartet von Berlin, daß hier die Literatur von morgen die Fühler ausstreckt. Die Atmosphäre und die Ost-West-Lage der Stadt begünstigen das Entstehen lebhafter Diskussionen, neuartiger literarischer Versuche, international recipierter Werke [...]. Vor allem junge Literaten, deutschsprachige und ausländische, fühlen sich von Berlin angezogen. Lesungen und Aufenthalte der San-Francisco Poets, der Autoren des roman nouveau, selbst der polnischen und jugoslawischen Avantgarde in Westberlin beweisen das.

Eine Stärkung Berlins könnte also gerade auf literarischem Gebiet erfolgreich sein. [...] Meines Erachtens sollte man Mittel zur Verfügung haben, um junge Literaten, deutsche und ausländische, nach Berlin einladen zu können. Sie sollten hier auf die Dauer von einem halben oder einem Jahr wohnen und an Colloquien teilnehmen, die für sie unter bestimmten Themenstellungen stattfinden. Daneben sollten sie aber genügend Zeit haben, mit der Stadt selbst in Kontakt zu kommen und ihre eigenen Arbeiten niederzuschreiben. ${ }^{24}$
\end{abstract}

Das war die Gründungsidee des Literarischen Colloquiums Berlin (LCB), des ersten Literaturhauses in Deutschland und sogar weltweit. Es ging damals - wie Höllerer deutlich macht - um die „Stärkung Berlins“, Hauptargument war die Stützung der West-Enklave im Osten. Und zugleich ging es um ein bestimmtes Programm und einen bestimmten Anspruch, nämlich die Moderne nach Deutschland zu holen, die Kunst, die als demokratisch, freiheitlich, aufgeklärt, innovativ, jung empfunden wurde:

Das Bewegende und Transitorische, in die Zukunft Hineinreichende wird in Berlin den Ausschlag für jeden einzelnen Programmpunkt geben müssen. Es muß sich nach der Einrichtung dieses literarischen Colloquiums herumsprechen, daß die jungen künstlerischen Bewegungen in Ost und in West hier in Berlin ein Forum und einen Fuchsbau haben, wie nirgend sonst in einer anderen Stadt! ${ }^{25}$

Auffällig ist die programmatische Fortschrittsorientierung, die Zukunftsausrichtung, es geht um die jungen künstlerischen Bewegungen; bemerkenswert erscheint aber auch die Wendung von „Forum“ und „Fuchsbau“: Berlin bietet demnach eine besondere Art und ein besonderes Maß an öffentlicher Aufmerksamkeit - und zugleich soll es künstlerische Zufluchtsstätte sein. Bedeutsam ist schließlich auch die Rede von West und Ost. Neben der westlichen Moderne sollte avantgardistische, experimentelle Literatur aus dem Osten eingeladen werden, jene Autoren und Autorinnen, die aus der Perspektive kulturpolitischer Dogmatiker im Osten als subversiv und dissidentisch einzustufen waren. „Durch Einladungen kommen Publikum und Autoren aus aller Welt mit Berlin in Kontakt; politische Wirkung literarischer Ereignisse!" ${ }^{26}$ so die programmatische Losung, der Höllerer seine Pläne unterstellte.

24 Walter Höllerer, Brief an Shepard Stone vom 27. Juli 1962. Nachlass Walter Höllerer, Literaturarchiv Sulzbach-Rosenberg, Signatur 03WH/DJ/A/4,7d (Hervorhebung im Text).

25 Ebd.

26 Ebd. (Hervorhebung im Text). In einem Brief an Shepard Stone vom 20. Dezember 1962, in dem Höllerer nochmals seine Pläne resümiert, betont er: „Das Programm soll in Berlin wirken und von Berlin ausstrahlen, es soll auch die Verbindung zwischen den Ländern jenseits des Eisernen Vorhangs 
Wie aus dem Brief an Stone hervorgeht, nahm das Projekt seinen Ausgang bei der Idee einer Creative Writing Schule, auch wenn die institutionellen Planungen von Beginn an darüber hinausgingen. Höllerer dachte dabei an die produktive Begegnung jüngerer mit arrivierten Autorinnen und Autoren, die sich grenzüberschreitenden ästhetischen Erfahrungen und Experimenten öffnen wollten: Es werde sich bei dem geplanten Colloquium ,ja um verschieden interessierte Gruppen handeln [...], z. T. um elementare Kurse in der ,Schule des Schreibens', z. T. um komplizierte KompositionsAuseinandersetzungen“. ${ }^{27}$ Im Archiv des LCB findet sich ein undatiertes, aber erkennbar sehr frühes Entwurfspapier, in dem Höllerer eine erste Kalkulation für das Colloquium festhält. Dort wird das gesamte Projekt schlicht mit „Creative Writing“ betitelt; darunter werden die Bereiche aufgeführt, in denen diese Schreibwerkstatt tätig werden sollte: „Roman, Drama, Lyrik, Hörspiel, Fernsehspiel, Essay, Kritik“. ${ }^{28}$ Das programmatische Stichwort des Creative Writing verweist auf die USA, wo Höllerer bei seinen diversen Aufenthalten und Gastprofessuren unter anderem in Harvard und Chicago schreibdidaktische Programme kennenlernen konnte. Seit den 1920er Jahren hatte sich an amerikanischen Colleges und Universitäten Creative Writing als eigenes Fach zu etablieren begonnen. In Deutschland waren derartige Kurse oder Studiengänge bis dahin unbekannt, ${ }^{29}$ und es war Höllerer, der diese Idee aus den USA nach Westberlin importierte. Offenbar war es gerade dieser Ansatz, der Shepard Stone besonders überzeugte. $^{30}$

1963 wurde dann das LCB gegründet mit einem Standort in Charlottenburg und einem zweiten in der Villa am Wannsee, die ab 1964 als Gästehaus diente und das Colloquium in seiner heutigen Form beherbergt. Die erste Creative

herstellen, z. B. mit Polen und Jugoslawien.“ Nachlass Walter Höllerer, Literaturarchiv SulzbachRosenberg, Signatur 03WH/DJ/A/4,12a.

27 Walter Höllerer, Brief an Shepard Stone vom 27. Juli 1962. Nachlass Walter Höllerer, Literaturarchiv Sulzbach-Rosenberg, Signatur 03WH/DJ/A/4,7d.

28 Nachlass Walter Höllerer, Literaturarchiv Sulzbach-Rosenberg, Signatur 03WH/DJ/A/2,10.

29 ,In Deutschland unbekannt‘ ist allerdings nicht ganz richtig, denn in der DDR gab es bereits eine, wenn auch sehr anders gelagerte, feststehende Institution der Schreibausbildung. 1955 war in Leipzig das Institut für Literatur gegründet worden, seit 1959 firmierte es unter dem Namen Institut für Literatur „Johannes R. Becher“. Es folgte dem Vorbild des 1933 in Moskau gegründeten Maxim-GorkiInstituts und bot ein reguläres Studium der Literatur an, das neben Veranstaltungen unter anderem zu Marxismus-Leninismus, Literaturgeschichte und Ästhetik auch ein Praktikum in Produktionsbetrieben vorsah - und sogenannte ,Schöpferische Seminare‘ für Prosa, Lyrik und Dramatik. Zur Geschichte des Instituts vgl. Isabelle Lehn, Sascha Macht, Katja Stopka: Schreiben lernen im Sozialismus. Das Institut für Literatur „Johannes R. Becher“, Göttingen 2018.

30 Brief von Walter Hasenclever an Walter Höllerer vom 17. Mai 1962: „Unser Vorschlag ist ihm [Shepard Stone] deshalb sympathisch, weil er darin eine Möglichkeit sieht, schöpferisch weiter zu wirken und eine Möglichkeit der Unterweisung zu schaffen, die es einfach sonst in Deutschland nicht gibt. Er würde unser Projekt sozusagen als den Prototyp alles dessen ansprechen, was ihm für die Berliner kulturelle Entwicklung (soweit sie die Ford Foundation interessiert) vorschwebt.“ Nachlass Walter Höllerer, Literaturarchiv Sulzbach-Rosenberg, Signatur 03WH/DJ/A/5,2. 
Writing-Veranstaltung fand von November 1963 bis Februar 1964 statt und widmete sich dem „Prosaschreiben“. Von Mai bis September 1964 folgte das Colloquium zum „Dramenschreiben“. Junge deutsche, englische und US-amerikanische Dramatiker und eine Dramatikerin - wohnten für mehrere Monate zusammen im Haus am Wannsee. Auch und gerade im Hinblick auf die ,Schreibschule‘ des Colloquiums zeigte sich also die Ambition, ein internationales Programm auf die Beine zu stellen: „Die Einladungen an die jungen Schriftsteller sollten nicht auf den deutschsprachigen Raum beschränkt bleiben“, ${ }^{31}$ hatte Höllerer bereits im Vorfeld gegenüber Stone nachdrücklich hervorgehoben. Erstaunlicherweise war dann der unter der Leitung von James Saunders stattfindende englischsprachige Dramenworkshop sogar erfolgreicher als der parallel dazu stattfindende deutschsprachige. Zumindest sah einer der Teilnehmer des deutschsprachigen Workshops dies so: Peter O. Chotjewitz veröffentlichte in der Studentenzeitschrift der FU unter dem Titel Aus der Schule geplaudert eine ziemlich heftige Kritik am LCB und konkret am deutschsprachigen Workshop, dessen Betreuung durch häufig wechselnde, zwar namhafte, aber nicht gerade theaterpraktisch ausgewiesene Literaten er als ungenügend empfand..$^{32}$ Auch die Sorglosigkeit im Geldausgeben wurde angeprangert, ein Punkt, der bald die Runde machen und zu ernsthaften Vorhaltungen seitens der Stiftung führen sollte, insofern das Literarische Colloquium bereits im Jahr 1964 einen Großteil des von der Ford Foundation zugesagten Geldes verausgabt hatte. ${ }^{33}$ Vielleicht lag es an den unbestreitbaren Schwierigkeiten, ein Creative Writing-Programm als freies Gespräch unter Interessierten zu organisieren, vielleicht an der angespannten finanziellen Lage, die ein derart üppiges Stipendienprogramm nicht hergab, oder am Unverständnis der deutschen, auf Genieästhetik abonnierten Öffentlichkeit, die Dieter E. Zimmer in der ZEIT für Schäden am Renommee der frisch gegründeten Institution verantwortlich machte: ${ }^{34}$ Das Format der Schreibwerkstätten wurde jedenfalls in der anfangs versuchten Gestalt nicht weitergeführt. Es folgten noch

31 Brief von Walter Höllerer an Shepard Stone vom 20. Dezember 1962. Nachlass Walter Höllerer, Literaturarchiv Sulzbach-Rosenberg, Signatur 03WH/DJ/A/4,12a.

32 Peter O. Chotjewitz: „Aus der Schule geplaudert“, in: Colloquium 9/10 (1964), 20-21.

33 Vgl. den Brief von Walter Hasenclever an Walter Höllerer vom 21. November 1964. Nachlass Walter Höllerer, Literaturarchiv Sulzbach-Rosenberg, Signatur 03WH/DJ/A/11,15.

34 „Soweit man in der Öffentlichkeit überhaupt irgendwelche Vorstellungen mit dem Literarischen Colloquium verbindet, denkt man sich eine Art Schreibschule für mehr oder minder begabte Literaturanwärter: Das Image der Institution wird ganz und gar von ihrer allerersten Unternehmung bestimmt, die ein viermonatiges Seminar unter dem Titel ,Prosaschreiben` war, zu dem im Winter 1963/64 vierzehn junge Leute, die hier und da etwas veröffentlicht hatten, eingeladen wurden. Die Idee, daß junge Autoren zu einer Art Kursus zusammenkommen, um sich von älteren Schreibaufgaben stellen zu lassen und ihre Erzeugnisse zu diskutieren, schien den Deutschen, die sich ihre Schriftsteller nur einzeln um die Inspiration ringend vorzustellen gewohnt sind, so abstrus, daß das Literarische Colloquium ein für allemal abgestempelt und seine weitere Tätigkeit verdunkelt war.“ Dieter E. Zimmer: „Die Literatur-Mafia von Berlin. Eine rabiate Polemik und einige nüchterne Beobachtungen“, in: Die Zeit Nr. 47 (18.11.1966). 
anders dimensionierte Colloquien zur Theater-, Lyrik- und Filmkritik (1965) und bezeichnend für den betont internationalen Zuschnitt des LCB - ein Colloquium zu „Problemen der Übersetzung“ (1966).

Ein Meilenstein im internationalen Einladungsprogramm Höllerers war die Veranstaltung Modernes Theater auf kleinen Bühnen. Für den Winter 1964/65 wurden Avantgarde-Ensembles aus Paris, Mailand, New York, London, Stockholm, Leningrad, Warschau, Belgrad, Zagreb und Mexico-City nach Berlin geholt. Auch hier wurden die Aufführungen im Fernsehen gezeigt; in diesem Fall übernahmen sie sogar alle deutschen Rundfunkanstalten, ein großer Erfolg, wie das Protokoll der Arbeitssitzung des Literarischen Colloquiums vom 26. März 1964 vermerkt: „Tatsächlich bedeuten also diese Veranstaltungen die erste große Publicity-Welle für das Literarische Colloquium, die auch nach dem Osten ausstrahlen wird." ${ }^{35}$ Schwieriger als die Ausstrahlung nach Osten gestaltete sich allerdings offenbar die Einladung von Ensembles aus dem Osten. So scheiterte beispielsweise der Versuch, ein experimentelles Theater aus Polen nach Berlin zu holen. Höllerer wandte sich in dieser Sache an den befreundeten polnischen Theaterwissenschaftler Andrej Wirth; dieser leitete die Einladung an das polnische Kulturministerium weiter. Im Mai 1964 übermittelte er die Absage des Ministeriums und erklärte die Ergebnislosigkeit seiner Bemühungen mit Schwierigkeiten „,geopolitischer Natur““: Der Grund liege ,in der unbequemen Lage der Stadt B. “36 Während es durchaus möglich sei, Einladungen polnischer Künstler in die Bundesrepublik zu realisieren, habe die Regierung kein Interesse, „offizielle kulturelle Beziehungen mit West-Berlin“37 einzugehen. Das „geopolitische“ Problem betraf die besondere Situation Berlins als Viersektorenstadt, deren rechtlicher Status in der Luft hing. Bis zum Viermächteabkommen von 1971, das de facto die Anerkennung der DDR durch die Westalliierten und die Bundesrepublik bedeutete, wurde die Bindung von Westberlin an die Bundesrepublik aus Ostperspektive als Provokation wahrgenommen, und immer wieder eine entsprechend restriktive Politik von den Ländern aus dem sowjetischen Herrschaftsbereich praktiziert.

Als weiteres literarisches Großereignis Höllerers sei die berühmte Reihe Ein Gedicht und sein Autor erwähnt, die Höllerer im Winter 1966/67 organisierte und die jeweils zwei Vertreter der internationalen Lyrikprominenz in der Westberliner Akademie der Künste zusammenbrachte, zu Lesung, poetologischem Statement und Diskussion, eine „Weltausstellung der Lyriker“38, wie die ZEIT titelte. Auch „dieses lyrische

35 Protokoll über die Arbeitssitzung des Literarischen Colloquiums am Donnerstag, den 26. März 1964. Nachlass Walter Höllerer, Literaturarchiv Sulzbach-Rosenberg, Signatur 03WH/DJ/A/12,8.

36 Brief von Andrej Wirth an Walter Höllerer vom 08. Mai 1964. Nachlass Walter Höllerer, Literaturarchiv Sulzbach-Rosenberg, Signatur 03WH/AA/3,17.

37 Brief von Andrej Wirth an Walter Höllerer vom 23. Mai 1964. Nachlass Walter Höllerer, Literaturarchiv Sulzbach-Rosenberg, Signatur 03WH/AA/3,17.

38 Dieter E. Zimmer: „Weltausstellung der Lyriker. Eine Veranstaltungsreihe des Literarischen Colloquiums Berlin“, in: Die Zeit Nr. 6 (10.02.1967). 
Marathon-Rennen von 21 Poeten an 10 Abenden“"39 war eine Fernsehveranstaltung, die im 3. Programm des SFB (Sender Freies Berlin) übertragen wurde. Eingeladen waren Gäste aus Schweden, Frankreich, Jugoslawien, Polen, Italien, USA, ČSSR, Österreich, DDR und UdSSR. Alle Eingeladenen kamen, nur einer nicht: „Günter Kunert, der am wenigsten weit entfernt - in Ostberlin - wohnende, konnte als einziger von den Eingeladenen nicht persönlich in der Akademie der Künste erscheinen“, ${ }^{40}$ wie Höllerer in seiner Einführung zum siebten Abend, an dem Miroslav Holub und Josef Hanzlik aus der ČSSR auftraten, betonte. Kunert war der Passierschein verweigert worden.

In der Neuen Zürcher Zeitung wurde - abseits der Ostberliner Restriktionen - das literarische Großereignis als „einleuchtendes Beispiel für die kulturelle Brückenfunktion Westberlins“ angeführt. Insbesondere der russisch-amerikanische Abend mit Andrej Wosnessenskij und Lawrence Ferlinghetti wurde gerühmt:

Ost und West am Vorlesepult, auf der Bühne in der Kunstgalerie - das sind die Ambitionen und Experimente im Berliner Kulturleben. Höllerer selber wünscht nicht nur Kontakte, Begegnungen und Festwochen; er möchte die Künstler zur Arbeit in Berlin und so zur Sesshaftigkeit animieren. Berlin als Atelier und Werkstätte, nicht nur als Schaufenster und Festsaal. ${ }^{41}$

\section{V.}

Über den Erfolg dieser Bemühungen konnte man natürlich streiten.

Im Dezember 1963 veröffentlichte die amerikanische Zeitschrift The Atlantic Monthly ein Gespräch zwischen Höllerer, Günter Grass und Walter Hasenclever über Writers in Berlin. In diesem Dreiergespräch gab es ein kleines Hin und Her über den kosmopolitischen Zuschnitt Berlins, d. h. Westberlins. Ähnlich wie Hans Werner Richter in dem eingangs zitierten Brief betont auch Höllerer, Berlin sei die einzige wirkliche Großstadt in Deutschland: „Berlin is probably the only city in Germany that can boast of an atmosphere which is genuinely metropolitan. The other cities are much more provincial.“ Dagegen äußert Grass entschiedene Zweifel: „I don’t know if you can call Berlin really metropolitan. The most provincial feature in Berlin is its hectic effort during the last two years to become a metropolis. This in itself is a sure sign of provincialism.“ " ${ }^{42}$ Gerade die forcierten Bemühungen um Internationalisierung auf

39 Manuskript der Sendung „Atelier am Sonntagabend“ vom 12. Februar 1967, RIAS, Hans Georg Soldat: Ein Gedicht und sein Autor. Höllerers Lyrik-Reihe des Literarischen Colloquiums. Nachlass Walter Höllerer, Literaturarchiv Sulzbach-Rosenberg, Signatur 03WH/BN/2,4 j, 6.

40 Walter Höllerer: [Einleitung zu Günter Kunert], in: ders. (Hrsg.): Ein Gedicht und sein Autor. Lyrik und Essay, München 1969, 220.

41 T. W.: „Westberlin - Bundesland und isolierte Metropole“, in: Neue Zürcher Zeitung, Fernausgabe Nr. 65 (07.03.1967), 3.

42 Höllerer, Grass, Hasenclever (Anm. 23), 111. 
Westberliner Seite, wie sie seit dem Mauerbau angestrengt wurden, nimmt Grass als Zeichen der Randständigkeit wahr.

Der Vorwurf des Provinzialismus Westberlins wurde unter anderen Vorzeichen auch an anderer Stelle geäußert: Im DSF-Journal, der Monatszeitung der Westberliner Gesellschaft für Deutsch-Sowjetische Freundschaft, wurden immer wieder Beispiele von Auftrittsverboten für Künstler aus sozialistischen Staaten angeführt. Unter der Überschrift Bonn macht Westberlin zur Provinz versammelte das DSF-Journal kritische Stimmen; Anlass war, dass es westdeutsche Behörden dem russischen Pianisten Swjatoslaw Richter untersagt hatten, in Westberlin zu spielen: Die Westberliner - so heißt es dort - „sollen in Zukunft möglichst nur noch das sehen, was von Ford oder dem Bundesverband der Industrie angeregt und finanziert wird.“43 - „Unsere Stadt wird immer mehr von der internationalen Kultur isoliert, zu der eben auch die weltbedeutenden Ereignisse der Sowjetunion und der anderen sozialistischen Länder gehören." ${ }^{44}$ Das war in der Tat ein Hauptanliegen dieses Journals: die Gegenperspektive gerade im Hinblick auf die internationale Bedeutung des Westberliner Kulturlebens geltend zu machen. Im Zentrum stand die Kritik an der „Frontstadt-Politik“45 und an dem, was ein Autor des Journals „Kulturquarantäne“46 oder „geistige Quarantäne“47 nannte: die Unterdrückung von künstlerischen Ereignissen oder kulturellen Veranstaltungen in Westberlin aufgrund ihrer Herkunft aus der Sowjetunion oder allgemeiner aufgrund ideologischer Vorbehalte. Es ist die Retourkutsche zum üblichen und sehr berechtigten - Vorwurf der Zensur Richtung Osten. Das DSF-Journal wollte jedenfalls deutlich machen, dass auch im Westteil der Stadt nicht unumschränkte Freiheit und Toleranz herrschten, womit es selbstverständlich auch, aber nicht nur als Sprachrohr der ostdeutschen Perspektive agierte.

\section{VI.}

Wie wurden nun die Westberliner Aktivitäten tatsächlich auf der anderen Seite der Mauer wahrgenommen? Ein Beitrag vom Februar 1963 aus der Zeitschrift Einheit zuständig für Theorie und Praxis des Wissenschaftlichen Sozialismus - mag die Ausgangslage im Osten veranschaulichen. Unter der Überschrift „Es gibt keine ideologische Koexistenz“ wird betont, dass gerade die deutsch-deutsche und insbesondere

43 Viktor Klaus, in: DSF. Zeitung für Frieden und Völkerverständigung 3/8 (1964), 4.

44 Ralf Elias, in: DSF. Zeitung für Frieden und Völkerverständigung 3/8 (1964), 4.

45 Wladimir Iwanow: „Frontstadt oder Freie Stadt“, in: DSF. Zeitung für Frieden und Völkerverständigung $1 / 9$ (1962), 3.

46 o. A.: „Gegen Kultur-Quarantäne“, in: DSF. Zeitung für Frieden und Völkerverständigung 1/2 (1962), 1.

47 Ebd. 
die Berliner Situation zu maximaler kulturpolitischer Entschiedenheit und zur Abwehr „kulturimperialistischer“ Tendenzen zwinge. Der Beitrag wendet sich gegen alle „Erscheinungen des Wiederauflebens formalistischer, modernistischer, abstrakter Kunstpraktiken“ ${ }^{48}$ in der DDR und fordert Parteilichkeit der Literatur mit Blick auf das sozialistische Menschenbild. Als Beispiel für eine Verwässerung des Klassenstandpunkts wird Jean-Paul Sartres Rede auf dem Weltfriedenskongress in Moskau 1962 angeführt, wo dieser die „Abrüstung der Kultur“ im Sinne einer transnationalen kulturellen Einheit gefordert hatte. ${ }^{49}$ Namen wie Peter Hacks oder Stephan Hermlin werden als Vertreter einer falschen modernistisch-formalistischen, schematischen Kunstauffassung erwähnt, weiterhin wird Peter Huchels Gestaltung der Zeitschrift Sinn und Form als Negativbeispiel genannt. Gegen die Berufung auf „Einzelerscheinungen im sowjetischen Kunstleben“ - wie beispielsweise die Lyrik Jewgeni Jewtuschenkos oder Andrej Wossnessenskis -, wird betont, dass diese von der Parteiführung der KPdSU entschieden zurückgewiesen worden seien. Entsprechend mahnt hier der ungenannte Beiträger:

Wir dürfen doch niemals übersehen, daß wir unsere sozialistische Nationalkultur im Angesicht eines erbittert gegen die gesetzmäßige sozialistische Entwicklung ankämpfenden imperialistischen Gegners aufbauen, der seit Jahrzehnten einen wütenden Antikommunismus verficht und heute alle Mittel der psychologischen Kriegsführung von Westdeutschland und von dem Störzentrum Westberlin aus gegen die DDR einsetzt. Gerade unsere Situation duldet kein Verwischen der ideologischen Gegensätze, keine „Aussöhnung“ der sozialistischen und der bürgerlichen Ideologie. [...] Ein Vergleich mit den hauptsächlichen Erscheinungen der Kulturleistungen der kapitalistischen Kunst, ihrem Zynismus, ihrer Perversion, ihrer das Menschenbild zerstörenden Tendenz unterstreicht, wie dringend es ist, keine „Abrüstung“ der sozialistischen Kunst zuzulassen..$^{50}$

Deutlich wird das Ausmaß, in dem Literatur und Kunst für den Kalten Krieg funktionalisiert werden. Dies gilt auch und erst recht für die internationale Kulturarbeit in Ostberlin in der ersten Hälfte der 1960er Jahre, die - mal direkt, mal indirekt - auf die internationalen Aktivitäten jenseits der Mauer bezogen ist. Nach dem Mauerbau mehren sich die Zeugnisse für neu erwachte bzw. mit neuem Elan vorangetriebene Internationalisierungsabsichten und -bemühungen auch im Ostteil der Stadt.

48 Es gibt keine ideologische Koexistenz. [Stellungnahme der „Einheit“, Heft 2 (Feb. 1963)], in: Elimar Schubbe (Hrsg.): Dokumente zur Kunst-, Literatur- und Kulturpolitik der SED, Stuttgart 1972, 822-824, hier: 822 (Dok. 259).

49 Vgl. Jean-Paul Sartre: Die Abrüstung der Kultur. Rede auf dem Weltfriedenskongreß in Moskau vom 09.-14.07.1962, in: ders.: Was kann Literatur? Interviews, Reden, Texte 1960-1976, hrsg. und mit einem Nachwort von Traugott König, Reinbek bei Hamburg 1979, 30-39. Die Rede wurde auch in der DDR veröffentlicht, in: Sinn und Form 5-6 (1962), 805-815.

50 Es gibt keine ideologische Koexistenz [Stellungnahme der „Einheit“, Heft 2 (Februar 1963)], in: Elimar Schubbe (Hrsg.): Dokumente (Anm. 48), 822-824, hier: 823 (Dok. 259). 
Die Internationalisierung wird dabei auch als Auseinandersetzung mit Westberlin und der bundesdeutschen Literatur dargestellt. Westberlin wird als „Pfahl im Fleische der Deutschen Demokratischen Republik“"51 und als „Frontstadt“52 in einem mit psychologischen Mitteln und kulturellen Waffen geführten Kampf wahrgenommen. Diese „Frontstadt-Politik“, so Wladimir Iwanow, Korrespondent der sowjetischen Nachrichtenagentur TASS in Westberlin, beruhe „auf der Ausnutzung Westberlins zur Wühltätigkeit gegen die DDR, in deren Zentrum es liegt. “53 Hiermit verbindet sich unmittelbar der politische Auftrag der literarischen Austauschbeziehungen nach dem Mauerbau. Ein Bericht über die Auslandsarbeit des Schriftstellerverbands der DDR im Jahr 1961 vermerkt folgendes:

\begin{abstract}
Die Hauptaufgabe unserer Delegationen, die nach dem 13. August ins Ausland fuhren, bestand darin, alle propagandistischen Möglichkeiten zu nutzen, um über das Deutschlandproblem und die Westberlin-Frage Klarheit zu schaffen. ${ }^{54}$

Bevor unsere Delegationen ins Ausland reisen, finden ausführliche Gespräche im DSV statt, an denen auch Mitarbeiter des Ministeriums für Auswärtige Angelegenheiten und des Ministeriums für Kultur teilnehmen. Die Kollegen werden über die politische und kulturelle Situation des gastgebenden Landes informiert und und [sic!] beauftragt alle entsprechenden Möglichkeiten zu nutzen, um über das Deutschlandproblem, den baldigen Abschluß eines Friedensvertrages, die Lösung der Westberlin-Frage und die Bändigujg [sic!] des westdeutschen Militarismus zu sprechen, bzw. zu schreiben..$^{55}$
\end{abstract}

Wendet man sich den internationalen literarischen Kontakten im Ostberlin der frühen 1960er Jahre zu, betritt man also unmittelbar die Bühne der auswärtigen Kulturpolitik. Dies hängt damit zusammen, dass Ostberlin nicht nur ein Teil von Großberlin war, sondern bekanntlich auch Hauptstadt der DDR, so dass hier zugleich der primäre Ort staatlicher Selbstdarstellung und außenkulturpolitischer Aktivitäten war. Vor allem aber hat es damit zu tun, dass in der DDR der 1950er und 1960er Jahre die auswärtige Kulturpolitik einen besonderen Stellenwert hatte, denn sie bildete bis zur offiziellen

51 Berlin - Kampffeld für den Frieden. Brief von Bodo Uhse an einen sowjetischen Poeten, in: Richter (Anm. 1), 81-83, hier: 81.

52 Hans Kochs Wortbeitrag, Das internationale Kolloquium des Deutschen Schriftstellerverbandes (DSV) „Die Existenz zweier deutscher Staaten und die Lage in der Literatur“, 1.-5. Dezember 1964 in Berlin (redigierte Tonbandabschrift), in: Elke Scherstjanoi (Hrsg.): Zwei Staaten, zwei Literaturen? Das internationale Kolloquium des Schriftstellerverbandes in der DDR, Dezember 1964, München 2008, 63.

53 Wladimir Iwanow: Frontstadt oder Freie Stadt, in: DSF. Zeitung für Frieden und Völkerverständigung, 1/9 (1962), 3.

54 Bericht über die Auslandsarbeit im zweiten Halbjahr (1961). Archiv des Schriftstellerverbandes der DDR, Literaturarchiv der Akademie der Künste Berlin (AdK), Signatur SV 276. Im Folgenden wird auf dieses Archiv mit der Sigle AdK verwiesen.

55 Bericht über die Auslandsarbeit seit Anfang August dieses Jahres (12. Oktober 1961). Archiv des Schriftstellerverbands der DDR, AdK, Signatur SV 276. 
völkerrechtlichen Anerkennung ein wichtiges Mittel der außenpolitischen Selbstdarstellung und Durchsetzung. ${ }^{56}$ Darüber hinaus ist die Dominanz der Sozialistischen Einheitspartei Deutschlands (SED) und die zentralistische Organisation aller gesellschaftlichen Bereiche unter Einschluss der Kultur verantwortlich dafür, dass bei den internationalen literarischen Kontakten, Austauschbeziehungen und Aktivitäten auch nicht-staatlicher Akteure das große Ganze, die Repräsentanz des Staates, die grundlegenden Ziele der außenpolitischen Selbstbehauptung und der internationalen staatlichen Anerkennung im Vordergrund standen.

Um nachvollziehen zu können, wie die außenkulturpolitischen Prozesse und im Speziellen die internationalen literarischen Kontakte abliefen, muss man sich die Zuständigkeiten für Kultur bzw. für internationale kulturelle Beziehungen im Staatsaufbau der DDR vor Augen führen. Man kann drei Ebenen unterscheiden: erstens die Parteiebene mit der SED-Führung und dem zentralen Apparat als der entscheidenden, Weisungen erteilenden Instanz der Macht, der gegenüber alle anderen berichtspflichtig waren. Involviert waren hierbei verschiedene Abteilungen des Zentralkomitees (ZK): für Kultur, für internationale Verbindungen und für Propaganda. Zweitens waren die staatlichen Organe beteiligt: das Ministerium für Auswärtige Angelegenheiten, das eine Kulturabteilung beherbergte und mitverantwortlich war für die Organisation des Kulturaustauschs mit anderen Ländern, sowie das Ministerium für Kultur, das seinerseits eine Abteilung für internationale Beziehungen hatte. Über die Aufgaben des Ministeriums für Kultur im Bereich der internationalen Beziehungen gibt die Verordnung über das Statut des Ministeriums für Kultur vom 21. November 1963 Auskunft: „Unterstützung aller ,humanistischen demokratischen Kräfte in Westdeutschland“; Kulturelle Zusammenarbeit mit anderen Völkern; Stärkung des internationalen Ansehens der DDR; Vorbereitung und Verwirklichung von Kulturabkommen und Kulturarbeitsplänen; Anleitung der Arbeit in internationalen Organisationen auf kulturellem Gebiet und der Auslandsarbeit der dem Ministerium für Kultur nachgeordneten Einrichtungen. “57

56 Vgl. Art. „Kulturpolitik“, in: Andreas Herbst, Winfried Ranke, Jürgen Winkler: So funktionierte die DDR. Lexikon der Organisationen und Institutionen, 552-566, hier: 564, in: Enzyklopädie der DDR. Personen, Institutionen und Strukturen in Politik, Wirtschaft, Justiz, Wissenschaft und Kultur Hrsg. v. Matthias Bertram. 2. Ausg., Berlin 2000 (Digitale Bibliothek Band 32), 8.851-8.880, hier: 8.876. Zu Entwicklung und Organisation der auswärtigen Kulturpolitik der DDR und ihrem Verhältnis zur BRD vgl. Olivia Griese: Auswärtige Kulturpolitik und Kalter Krieg. Die Konkurrenz von Bundesrepublik und DDR in Finnland 1949-1973, Wiesbaden 2006, bes. 34-54.

57 Gesetzblatt der Deutschen Demokratischen Republik, Teil II, Nr. 110 vom 29. Dezember 1963: Verordnung über das Statut des Ministeriums für Kultur vom 21. November 1963. Stiftung Archiv der Parteien und Massenorganisationen der DDR, Bundesarchiv (BArch), Signatur ZB 20049 a/110, zit. nach Katrin Jäcke, Johanna Marschall-Reiser: Ministerium für Kultur. Teil 4: Hauptabteilung Internationale Beziehungen/DR 1. Einleitung zum Online-Findbuch ARGUS im Bundesarchiv, Berlin 2010. Online abgerufen am 27. März 2021 auf ARGUS im Bundesarchiv unter http://www.argus.bstu.bundesarchiv. de/dr1_ib/index.htm?kid=66BCAF39B0B542B99223F9DC7BE9E7C2. 
Neben der Partei und den Ministerien gab es drittens nicht-staatliche bzw. gesellschaftliche Organisationen wie den Kulturbund, ${ }^{58}$ die Akademie der Künste (Ost), die Liga für Völkerfreundschaft oder den Deutschen Schriftstellerverband (DSV). Letzterer ist als Berufsverband der Autor:innen in der DDR für unseren Zusammenhang besonders wichtig; innerhalb des DSV gab es ebenfalls eine eigene Auslandsabteilung, die sich um internationale Beziehungen bemühte. Darüber hinaus beteiligte sich der DSV an internationalen Autorenverbänden: an der COMES (Comunitá europea degli scrittori), einer 1958 gegründeten, linksgerichteten europäischen Schriftstellervereinigung, ${ }^{59}$ sowie dem internationalen PEN. Auch hier, im Verhältnis der nicht-staatlichen Organisationen zu Partei und Ministerien, galt das zentralistische hierarchische Prinzip, das heißt auch der Schriftstellerverband war berichtspflichtig und den Weisungen der Partei bzw. der staatlichen Stellen unterstellt. Dennoch zeigte sich zwischen den beteiligten Organisationen und Akteuren ein Ineinander unterschiedlicher Ansätze und Interessen, so dass es durchaus zu Konflikten des DSV mit zentralen Vorgaben der Partei oder der staatlichen Stellen kam.

\section{VII.}

Die internationalen kulturellen Kontakte im Rahmen der nicht-staatlichen Organisationen verliefen auf unterschiedlichen Gleisen. Das wichtigste Instrument der internationalen Kulturarbeit waren die sogenannten Freundschaftsgesellschaften, die beginnend bereits Ende der 40er Jahre - nach sowjetischem Vorbild etabliert wurden. Ab 1961 waren sie beheimatet unter dem Dach der Liga für Völkerfreundschaft, „deren

58 Der Kulturbund war besonders eng an der SED gebunden, vgl. Brigitte Fischer: Kulturbund/DY 27, Einleitung zum Online-Findbuch ARGUS im Bundesarchiv, Berlin 2012. Online abgerufen am 27. März 2021 auf ARGUS im Bundesarchiv unter http://www.argus.bstu.bundesarchiv.de/dy27/index. htm?kid=020865c8-2c24-4def-bfc4-971d857c4116; vgl. Andreas Zimmer: Der Kulturbund in der SBZ und in der DDR. Eine ostdeutsche Kulturvereinigung im Wandel der Zeit zwischen 1945 und 1990, Wiesbaden 2018. Online unter: https://link.springer.com/book/10.1007/978-3-658-23553-6.

59 Die COMES vereinte Mitglieder aus west- und osteuropäischen Ländern. In den Diskussionen des DSV spielt die COMES und die Teilnahme von Schriftstellern aus der DDR an deren Treffen durchaus eine gewisse Rolle. In einem sehr umfangreichen „Entwurf einer Konzeption der internationalen Arbeit des Schriftstellerverbandes“ (wohl aus dem Jahr 1967) wird eine stärkere Berücksichtigung der COMES eingefordert: „Die Möglichkeiten, die internationalen Organisationen der Literaturschaffenden in die internationale Arbeit des DSV einzubeziehen, sind noch nicht im genügenden Maße genutzt. Die jeweils zuständigen Aktivs des DSV werden sich gemeinsam mit der Auslandskommission und der Auslandsabteilung intensiver mit unserer Arbeit in folgenden Organisationen beschäftigen: ,Comes“ / ,Internationale Übersetzungsorganisation“ / ,Internationale Kritikerorganisation““. Entwurf einer Konzeption der internationalen Arbeit des Deutschen Schriftstellerverbandes. Archiv des Schriftstellerverbands der DDR, AdK, Signatur SV 154, Bl. 61. Vgl. auch Hans Koch: Zu einigen Fragen unserer Auslandsarbeit. in: Neue Deutsche Literatur 5 (1964), 153-163, hier: 153. 
direkte Abhängigkeit vom ZK im Ausland kaum bekannt war“, ${ }^{60}$ wie Frank Trommler in seiner Studie über die deutsche auswärtige Kulturpolitik im 20. Jahrhundert betont. Eine herausgehobene Rolle spielte hierbei naheliegenderweise die Deutsch-Sowjetische Freundschaftsgesellschaft, die 1947 in Gesamtdeutschland gegründet wurde, in nahezu allen westdeutschen Bundesländern bald verboten, in der DDR aber intensiv betrieben wurde und - wie schon kurz erwähnt ${ }^{61}$ - in Westberlin bis zum Mauerfall überlebte. ${ }^{62}$

Allerdings konnte es auch vermittelt über Freundschaftsgesellschaften durchaus interessante und nicht primär von außenkulturpolitischen Erwägungen getragene literarische Begegnungen geben, wie das Beispiel einer deutsch-deutsch-sowjetischen Begegnung auf Westberliner Boden zeigt:

Im April 1964 war der sowjetische Autor Konstantin Simonow als Gast der Gesellschaft für Deutsch-Sowjetische Freundschaft in Ostberlin, um Material für den dritten Teil eines Romanzyklus über den Zweiten Weltkrieg zu sammeln. Simonow war im Zweiten Weltkrieg als Kriegsberichterstatter tätig gewesen, er hatte Lobgesänge auf Stalin verfasst, aber auch sehr populäre Liebesgedichte. Als sein Hauptwerk gilt die Romantrilogie, die den Kampf der Roten Armee gegen die deutsche Wehrmacht, aber auch Fehler der sowjetischen Führung um Stalin behandelt. In Ostberlin recherchierte Simonow nicht nur für seinen Roman, er nahm auch ,an einer Diskussion über seine Romane im ,Club der Kulturschaffenden' teil, und er war einige Stunden Gast der Bitterfelder Konferenz“", ${ }^{63}$ wie im Westberliner $D S F$-Journal vermerkt wurde.

Während seines Berlin-Aufenthalts suchte Simonow das Gespräch mit Günter Grass, der als Autor der Blechtrommel und politisch präsenter Schriftsteller einen außerordentlichen Ruf genoss; das Gespräch wurde über die Deutsch-Sowjetische Freundschaftsgesellschaft Westberlin in die Wege geleitet. ${ }^{64}$ Grass stellte allerdings die Bedingung, dass Uwe Johnson, der 1959 aus der DDR nach Westberlin übergesiedelt war, an dem Gespräch beteiligt werden sollte. Johnson war persona non grata im Osten;

60 Frank Trommler: Kulturmacht ohne Kompass. Deutsche auswärtige Kulturbeziehungen im 20. Jahrhundert, Köln 2013, 667.

61 Vgl. Abschnitt V.

62 Zum komplexen Ineinander sowjetisch-westlicher Freundschaftsgesellschaften über den gesamten Zeitraum des Kalten Krieges hinweg (mit Schwerpunkt auf England und Frankreich und unter Ausschluss von Westberlin) vgl. Sonja Großmann: Falsche Freunde im Kalten Krieg. Sowjetische Freundschaftsgesellschaften in Westeuropa als Instrumente und Akteure der Cultural Diplomacy, Berlin/Boston 2019.

63 Begegnung Westberliner Schriftsteller mit Konstantin Simonow, in: DSF. Zeitung für Frieden und Völkerverständigung 3/5 (1964), unpag.

64 Vgl. hierzu die Ausführungen von Klaus Völker im Interview mit Jutta Müller-Tamm und Ingo Schulze vom 24. Juni 2020, Mitschnitt einsehbar online auf der Homepage des EXC 2020 Temporal Communites (FU Berlin). Online abgerufen am 27. März 2021 unter https://www.temporal-communities.de/explore/listen-read-watch/lectures/berliner-weltliteraturen/videos-weltliteraturen/interviewvoelker-lang/index.html. 
so hatte er beispielsweise zu dem internationalen Schriftsteller-Kongress, der 1963, also im Jahr zuvor, in Leningrad stattgefunden hatte und an dem Hans Werner Richter und Hans Magnus Enzensberger von westdeutscher Seite aus teilgenommen hatten, kein Einreisevisum von den sowjetischen Behörden erhalten. ${ }^{65}$

Thema des Gesprächs zwischen Simonow, Grass und Johnson ist die Übersetzungs- und Publikationspraxis in der Sowjetunion. Diskutiert wird die Ablehnung von Boris Pasternaks Roman Doktor Schiwago, für dessen Nicht-Erscheinen in der Sowjetunion Simonow verantwortlich war; diskutiert wird weiterhin das Beispiel von Kafka, dessen Veröffentlichung oder Nicht-Veröffentlichung in den Ostblock-Staaten zu den virulentesten literaturpolitischen Fragen der früheren 1960er Jahre gehörte. Johnson und Grass monieren die „altmodischen Erzählweisen“66 der russischen Prosaliteratur, wenden sich gegen die Doktrin des sozialistischen Realismus und gegen die Zensurpraxis in der UdSSR. Zuletzt macht Grass den mit besonderem Ernst dargelegten, aber offenkundig nicht so gemeinten Vorschlag einer Reprivatisierung der Verlage in der Sowjetunion - das heißt: nur der Verlage -, damit ein aus ideologischen Gründen abgelehnter Autor noch die Chance hätte, sein Werk zu publizieren.

Das Gespräch wurde für das Spandauer Volksblatt geführt, aber mehrfach abgedruckt, u. a. in der linksgerichteten internationalen Presse. Das Neue Deutschland, die Tageszeitung der SED, und das Journal der Deutsch-Sowjetischen Freundschaft Westberlin, die doch eigentlich ein großes Interesse hätte zeigen müssen, brachten hingegen nur jeweils eine kurze Meldung: wohl weil das Gespräch zu kritisch für die Sowjetunion ausfiel. ${ }^{67}$

Dennoch berührt dieses Gespräch einen Punkt von allgemeinerer Bedeutung, denn es ist charakteristisch für die Konjunktur des Dialogformats in dieser Zeit. Zu den früheren 1960er Jahren gehören auch die internationalen Gespräche zwischen Kulturschaffenden, die Versuche, die Mauer im Dialog zu überwinden. Die Schriftsteller-Gespräche und literarischen Ost-West-Dialoge dieser Zeit sind gewissermaßen die andere Seite der Medaille, auf deren vorderer Seite der kulturelle Kalte Krieg steht. Wo ideologische Abgrenzung und politische Selbstbehauptung mit den Mitteln der

65 Zur COMES-Tagung in Leningrad 1963 vgl. Tanja Walenski: Verweigerte Entstalinisierung. Die Beziehungen des ,Literatursystems DDR` zur Sowjetunion, 1961-1989, Frankfurt a. M. u. a. 2009, 67-75, bes. 75 .

66 „Übersetzen - und kennenlernen (Gespräch zwischen Grass - Johnson - Simonow)“, in: Spandauer Volksblatt, Nr. 5449 (03.05.1964), 18. Zum Versuch, das Spandauer Volksblatt als überregionale Zeitung gegen die Springer-Presse zu positionieren, und zu Uwe Johnsons und Günter Grass' Unterstützung dieses Versuchs vgl. Uwe Johnson, Anna Grass, Günter Grass: Der Briefwechsel, hrsg. von Arno Barnert, Frankfurt a. M. 2007, 38-47.

67 Le Figaro Littéraire (10.06.1964), 3; Il Giorno (18.06.1964), 9; „Gespräch in Westberlin“ in: Neues Deutschland Nr. 120 (01.05.1964), 4; DSF. Zeitung für Frieden und Völkerverständigung 3/5 (1964), unpag. Eine maschinenschriftliche Abschrift des Gesprächs aus dem Spandauer Volksblatt findet sich im Archiv des Schriftstellerverbandes der DDR, AdK, Signatur SV 877, Bl. 192-198. 
Kultur vorherrschen, werden umgekehrt die Bemühungen um Verständigung geweckt, die transnationalen Begegnungsversuche, die grenzüberschreitenden, weltenumspannenden Dialoge - und die Vorstellung, dass gerade Literatur und Kultur berufen seien, über die Gräben der politischen Systeme hinweg Verständigung zu erzielen. Eben dies hatte Sartre gemeint, als er 1962 gegen den „kulturellen Bellizismus“, gegen die politische Mobilisierung der Kultur ihre Abrüstung und die „Vereinigung aller Kulturschaffenden“ forderte. ${ }^{68}$ Aber natürlich ließ sich auch dieses Dialogmodell wieder instrumentalisieren - denn beide Seiten stellten sich gern als friedliebend internationalisierend, die andere hingegen als propagandistisch ausgreifend dar.

\section{VIII.}

Die wichtigste nicht-staatliche Institution im Bereich der Literatur war der Schriftstellerverband der DDR, gegründet 1950 auf Betreiben der SED als Deutscher Schriftstellerverband (DSV). Der DSV war zwar die Berufsorganisation der Schriftsteller:innen in der DDR, handelte aber, wie alle Massenorganisationen, in Übereinstimmung mit den Vorgaben des ZK der SED und - insbesondere was die Auslandsarbeit betrifft - in enger Abstimmung mit den beiden genannten Ministerien. ${ }^{69}$ Präsidentin war von 1952 bis 1978 Anna Seghers. Bert Brecht und Stefan Heym gehörten in den 1950er Jahren dem Vorstand an, im Lauf der Zeit wurden aber vor allem linientreue Kulturfunktionäre in den Vorstand und die Ämter des DSV gesetzt.

Das zentrale Mittel der Auslandsarbeit waren auch für den DSV sogenannte Freundschaftsverträge, also Kooperationsvereinbarungen mit den Bruderverbänden in anderen sozialistischen Ländern; ${ }^{70}$ sie bildeten die institutionelle Basis für die internationale Arbeit. Im Jahr 1964 bestanden beispielsweise Freundschaftsabkommen zu

68 Sartre (Anm. 49), 36, 38.

69 Zum DSV vgl. Sabine Pamperrien: Versuch am untauglichen Objekt. Der Schriftstellerverband der DDR im Dienst der sozialistischen Ideologie, Frankfurt a. M. 2004; Thomas William Goldstein: Writing in Red. The East German Writers Union and the Role of Literary Intellectuals in the German Democratic Republic, 1971-90, Chapel Hill, N. C. 2010.

70 Später wurden auch Kooperationsvereinbarungen mit Verbänden nicht-sozialistischer Länder geschlossen. So heißt es beispielsweise in einer Auswertung des Internationalen Schriftstellertreffens in Berlin und Weimar von 1965: „Mit den offiziellen Vertretern vieler Schriftstellerorganisationen wurden Verbindungen angeknüpft. Wir nennen hier nur Griechenland, Dänemark, Zypern, Irak, Japan und eine Reihe lateinamerikanischer Länder. Es wurden Verhandlungen angebahnt, die zum Abschluß von Freundschaftsverträgen mit den entsprechenden Schriftstellerorganisationen führen sollen. Mit dem Verband Uruguays ist ein solcher Vertrag bereits unterschrieben worden. In der nächsten Zeit erwarten wir eine Delegation japanischer Schriftsteller, Schriftsteller aus der DDR werden Japan besuchen." Bericht des Sekretariats an den Vorstand. Archiv des Schriftstellerverbandes der DDR, AdK, Signatur SV 152. 
den Verbänden der UdSSR, der ČSSR, den Volksrepubliken Polen, Ungarn, Rumänien und Bulgarien sowie der Sozialistischen Föderativen Republik Jugoslawien. Mit Kuba und der Mongolischen Volksrepublik war man in Verhandlungen. ${ }^{71}$

Derartige Verträge wurden in der Regel für eine Dauer von ein oder später zwei Jahren abgeschlossen, und dann immer wieder neu vereinbart. Dieser Rhythmus hing damit zusammen, dass es tatsächlich um konkrete Planungen ging: Es gab eine vertraglich festgelegte Aufenthaltsquote, das heißt es wurde bestimmt, wie viele Personen für welche Dauer in das jeweils andere Land reisen sollten, und welche Seite dafür die Kosten zu tragen hatte. Verschiedentlich wurde zu Beginn der 1960er Jahren die bisherige Praxis kritisiert - es handele sich um „reine Routineverträge “, ${ }^{72}$ die nicht mit der nötigen Sorgfalt realisiert worden seien, so heißt es in einem Arbeitsplan des DSV für das erste Halbjahr 1962; und zwei Jahre später betonte Hans Koch, der Erste Sekretär des DSV, es sei notwendig „den Übergang vom Touristenaustausch zu echten Arbeitsdelegationen zu sichern und internationale Kolloquien, Seminare usw. zur inhaltlichen Hauptform der Arbeit mit ausländischen Delegationen zu machen.“73

Das Prinzip dieser Austauschbeziehungen waren also nicht Einladungen, sondern Entsendungen; das heißt: Das jeweils andere Land wählte aus, wer in die DDR reiste und ebenso umgekehrt. Es wurden Delegationen geschickt, nicht Einladungen ausgesprochen. Das machte den Schriftstelleraustausch zu einer heiklen diplomatischen Angelegenheit, denn man achtete in den Empfängerländern genau darauf, ob bedeutende oder weniger bedeutende Schriftsteller:innen entsandt wurden, und leitete daraus ab, welchen Stellenwert man selbst in den entsendenden Ländern hatte. $\mathrm{Zu}$ jung, zu unbedeutend, zu unbekannt: das kam einer Beleidigung der Zielländer gleich. In den Akten des Schriftstellerverbandes ist verschiedentlich die Rede von den Problemen, repräsentative und ausreichend geschulte Personen ins Ausland schicken zu können:

Nach wie vor gibt es gewisse Schwierigkeiten in der Zusammenstellung unserer Delegationen, weil die befreundeten Verbände einerseits repräsentative Vertreter unserer Literatur wünschen, andererseits aber auch nicht nur ältere, sondern auch jüngere Schriftsteller immer wieder, und zum Teil sehr kurzfristig, absagen. Vielleicht läßt sich diesbezüglich die Lage bessern, wenn wir dem Vorschlag unserer sowjetischen Freunde folgen und Genre-Delegationen schicken und empfangen, die für die literarische Arbeit der betreffenden ergiebiger sind. Aber wir dürfen auch nicht vergessen, daß die Delegationsreisen nicht nur Studienzwecken dienen, sondern

71 Koch (Anm. 59), 155 f. Eine „Sekretariatsvorlage“ der Auslandsabteilung vom 14. November 1964 enthält eine Bestandsaufnahme der Auslandsbeziehungen, gegliedert in „Sozialistisches Ausland“, „Kapitalistisches Ausland, Nationalstaaten und Republik Kuba“ und „Westdeutsche Arbeit“ unter Verweis auch auf Westberlin. Archiv des Schriftstellerverbandes der DDR, AdK, Signatur SV 147, Bl. 1-5. 72 Entwurf: Arbeitsplan für das erste Halbjahr 1962. Archiv des Schriftstellerverbandes der DDR, AdK, Signatur SV 276.

73 Koch (Anm. 59), 155. 
daß damit mehr denn je ein politischer Auftrag verbunden ist, nämlich das Ansehen der DDR allseitig zu heben. ${ }^{74}$

Offensichtlich war man aber mit dieser starren Praxis des internationalen Austauschs im DSV unzufrieden, wie beispielsweise ein Protokoll der Vorbereitungssitzung für das internationale Schriftstellertreffen 1965 erweist, in der Paul Wiens - seit 1961 Vorsitzender des Bezirksverbands Berlin im DSV - Unverständnis äußert über die strenge Einhaltung dieser Entsendungspolitik. ${ }^{75}$

\section{IX.}

Im Folgenden sollen anhand einer Episode die Problematik internationaler Einladungen jenseits der offiziellen Freundschaftsverträge, die Rolle einzelner Akteur:innen oder Mittler:innen darin und die unmittelbare Konkurrenz, die in den internationalen Beziehungen zwischen beiden Teilen der Stadt herrschte, erläutert werden. Das Beispiel betrifft die Einladung des sowjetischen Lyrikers Jewgeni Jewtuschenko nach Ostberlin im Jahr 1962. ${ }^{76}$ Jewtuschenko war zu Beginn der 1960er Jahre eine Kultfigur des internationalen Literaturbetriebs und galt als der zornige junge Mann der sowjetischen Lyrik. Berühmt wurde er 1961 mit seinem Gedicht Babi Jar, das an den deutschen Massenmord an Kiewer Juden 1941 erinnert und antisemitische Tendenzen in der Sowjetunion thematisiert. In den frühen 1960er Jahren war er nicht nur in der UdSSR ein Star, dessen Auftritte Stadien füllten, er tourte auch sehr erfolgreich durch Europa und die Welt, immer begleitet von einer großen publizistischen Resonanz. ${ }^{77}$

Folgen wir der Chronologie der Ereignisse: Im Sommer 1962 soll im Berliner Verlag Volk und Welt - der DDR-Verlag für internationale Literatur - Jewgeni Jewtuschenkos Gedichtband Mit mir ist folgendes geschehen erscheinen. ${ }^{78}$ Der Verlag will gemeinsam

74 Bericht für die Vorstandssitzung am 20. September 1962 (Ref.: Otto Braun). Archiv des Schriftstellerverbandes der DDR, AdK, Signatur SV 129.

75 Vgl. das Protokoll der erweiterten Vorstandssitzung im DSV vom 08. Januar 1965. „Gen. Eckert: Auf Grund der Freundschaftsverträge mit den Verbänden im sozialistischen Ausland können wir keine einzelnen Einladungen in diese Länder schicken, sondern es bestimmen die dortigen Verbände, welche Schriftsteller sie entsenden. [...] P. Wiens: Es ist unverständlich, warum die Form des Einladens unter den sozialistischen Ländern so streng gewahrt wird. Einige unserer Schriftsteller könnten doch persönliche Einladungen an Kollegen dort aussprechen." Archiv des Schriftstellerverbandes der DDR, AdK, Signatur SV 359/2, Bl. 86.

76 Der Vorgang wurde - mit einer etwas anderen Akzentsetzung - rekonstruiert von Walenski (Anm. 65), 37-41.

77 Vgl. „Sowjet-Poet Jewtuschenko“ als Titelbild in: Der Spiegel 22 (1962).

78 Jewgeni Jewtuschenko: Mit mir ist folgendes geschehen. Gedichte in Russisch und Deutsch. Ausgewählt, eingeleitet und aus dem Russisch übers. von Franz Leschnitzer, Berlin 1962. 
mit der Erfurter Zeitung Das Volk eine Dichterlesung mit Jewtuschenko organisieren, beide haben keine oder jedenfalls nicht ausreichende Mittel für eine derartige Einladung; ein Brief geht an Franz Leschnitzer, Jewtuschenkos Übersetzer, mit der Bitte um Rat. Leschnitzer ruft daraufhin in Berlin beim Schriftstellerverband an und fragt nach den Möglichkeiten, Jewtuschenko einzuladen. Der Verband gibt die Auskunft, er habe keine Möglichkeit, „für den Schriftstelleraustausch im Rahmen des Kulturabkommens persönliche Einladungen ergehen zu lassen und habe auch keine Mittel für irgendwelche persönliche Einladungen außerhalb des Kulturabkommens ““. ${ }^{79}$ Eine derartige Einladung könne nur über das Kulturministerium erfolgen. Der Verlag Volk und Welt bringt allerdings noch die Möglichkeit ins Spiel, dass die Gesellschaft für Deutsch-Sowjetische Freundschaft oder der Berliner Magistrat im Rahmen der Berliner Festtage Jewtuschenko einladen könnte. ${ }^{80}$

Man sieht - das gilt natürlich auf beiden Seiten der Mauer - die Einladung internationaler Schriftsteller ist zunächst eine Sache der Finanzen, und in der DDR fehlen die Mittel. Sodann muss die Einladung in geregelte Bahnen geleitet, in institutionelle Zusammenhänge eingespeist werden: Staatliche Stellen, wie das Kulturministerium oder der Berliner Magistrat, und Massenorganisationen, wie der Schriftstellerverband oder die Deutsch-Sowjetische Freundschaft, werden aufgerufen; diese sind allerdings auch an die im Rahmen der Freundschaftsverträge und Kulturabkommen vereinbarten Austauschprogramme gebunden. Das Prinzip dieser Austauschbeziehungen sah, wie gezeigt, individuelle Einladungen nur als Ausnahme vor. ${ }^{81}$

79 Aktennotiz, Berlin, 16.07.1962. Archiv des Schriftstellerverbandes der DDR, AdK, Signatur SV 873, Bl. 176.

80 Im August ergeht nochmals die schriftliche Anfrage an die Auslandsabteilung des DSV, Mitte August die endgültige Absage unter Verweis darauf, dass man bereits Galina Nikolajewa über den Rahmen des Freundschaftsvertrags hinaus eingeladen habe und damit das Finanzvolumen erschöpft sei. In einer Aktennotiz von 14. September 1962 ist festgehalten, dass der DSV auf Anfrage des Magistrats die Auskunft gab, dass „,der DSV nichts tun könnte, da wir dem Sowjetischen Verband nicht vorschreiben könnten wen sie delegieren.“ Archiv des Schriftstellerverbandes der DDR, AdK, Signatur SV 873/2. 81 Mit welchem diplomatischen Aufwand individuelle Einladungen des DSV verbunden sein konnten, zeigt der Vorgang um die erfolgreiche Einladung von Michail Scholochow, Verfasser des Romans Der stillen Don, für den er 1965, also zwei Jahre später, den Nobelpreis erhalten sollte. In einer Aktennotiz über ein Gespräch zur Vorbereitung des Freundschaftsvertrags zwischen den Schriftstellerverbänden der UdSSR und der DDR am 15. August 1963 wird zuletzt, nachdem die Fragen rund um den Austausch von Delegationen geklärt sind, in einem eigenen Punkt der Wunsch nach einer einzelnen Einladung angeführt: „Es wurde über die Möglichkeit einer Einladung von Genossen Scholochow in die DDR gesprochen. Genosse Markow sichert zu, dass der sowjetische Verband seinerseits an Genossen Scholochow herantreten wird, wenn eine Einladung über die Zentralkomitees der Parteien erfolgt.“ Aktennotiz über eine Zusammenkunft im Haus der Schriftsteller, Moskau, am 15. August 1963. Archiv des Schriftstellerverbandes der DDR, AdK, Signatur SV 873/2. Scholochow wird tatsächlich 1964 in der Einladungskartei des DSV geführt, er war aber zugleich Gast des Zentralkomitees der SED; sein Aufenthalt wurde mit großem Aufwand vorbereitet und war mit einem ausgefeilten Programm versehen, an dem auch der DSV massiv beteiligt war. Vgl. die umfangreiche Dokumentation im Archiv des Schriftstellerverbandes der DDR, AdK, Signatur SV 885. 
Damit allerdings sind die Bemühungen um Jewtuschenko noch nicht ans Ende gekommen. Im Juli schaltet sich Stefan Heym ein und schlägt Jewtuschenko als Eröffnungsgast einer internationalen Veranstaltungsreihe vor. ${ }^{82}$ Mitte September ruft Franz Leschnitzer erneut beim Schriftstellerverband an mit der Nachricht, dass es nun zu spät für eine Einladung sei, „da J. Jewtuschenko für zwei Monate nach Kuba fährt, anschließend Italien und Westdeutschland besucht." ${ }^{\text {83 }}$ In der Tat hielt sich Jewtuschenko zur Zeit der Kuba-Krise dort auf. Wegen der akuten Konfrontation zwischen den USA und der UdSSR kehrte er allerdings auch vorzeitig zurück - und im Bemühen, bei der Einladung des Stars die Nase vorn zu haben, melden sich wieder die Ostberliner zu Wort. In dem Telefonat mit dem Schriftstellerverband hatte Leschnitzer erwähnt, daß Jewtuschenko „,aus Westdeutschland allein 12 Einladungen erhalten“ 84 habe. Ostberlin will aber im Kampf der Internationalisierung nicht gegenüber dem Westen zurückstehen. Am 22. November 1962 wendet sich Otto Braun, Erster Sekretär des DSV, an Woronkow, den geschäftsführenden Sekretär des Schriftstellerverbandes der UdSSR:

Durch Zufall haben wir erfahren, daß Jewgeni Jewtuschenko jetzt schon von Kuba nach Europa zurückkehrt. Wir möchten diese Gelegenheit benutzen, um ihn in die DDR einzuladen und ihn bitten, in Berlin - selbstverständlich nicht Westberlin, sondern in unserer Hauptstadt - eine Lesung bzw. ein Forum durchzuführen.

Ich habe darüber schon vor längerer Zeit mit unserem Freund Konstantin Fedin korrespondiert, und er wollte mit Ihnen und Genossen Surkow darüber sprechen.

Es liegt uns sehr viel daran, daß Jewgeni Jewtuschenko, bevor er evtl. in Westdeutschland auftritt, zuerst in unserer Deutschen Demokratischen Republik spricht. Die Gründe dafür liegen auf der Hand. ${ }^{85}$

Der sowjetische Starpoet soll nicht vom Westen vereinnahmt werden. Aber auch diese Bemühungen, Jewtuschenko zuerst - vor allen zwölf westdeutschen Einladungen in Ostberlin zu haben, scheitern. Unter größter Aufmerksamkeit der Presse tourt Jewtuschenko dann im Januar 1963 durch die BRD, wo er als Idol der poststalinistischen Lyrik gefeiert wird.

82 Vgl. hierzu unten Abschnitt XII.

83 Archiv des Schriftstellerverbandes der DDR, AdK, Signatur SV 873, Bl. 173.

84 Ebd.

85 Archiv des Schriftstellerverbandes der DDR, AdK, Signatur SV 873. 


\section{$\mathbf{X}$}

Über die vom DSV organisierten Aufenthalte ausländischer Schriftsteller und Schriftstellerinnen in der DDR informiert die Einreisekartei im Archiv des Schriftstellerverbandes. ${ }^{86}$ Punktuelle Ausschläge im Gesamtvolumen der Einreisevorgänge hängen mit größeren Veranstaltungen zusammen, die der Schriftstellerverband organisierte, so 1964 das Internationale Kolloquium des Schriftstellerbands in Berlin, das Teilnehmer aus sechs sozialistischen Staaten zusammenführte, oder 1965 das Internationale Schriftstellertreffen in Berlin und Weimar, das Teilnehmer aus sozialistischen und nichtsozialistischen Ländern vereinte. Gerade das Kolloquium von 1964 ist ein Beispiel dafür, dass die kulturpolitischen Frontlinien in der internationalen Begegnung nicht nur gegen den Westen verliefen, sondern dass Reibungen und Verwerfungen auch innerhalb des sozialistischen Lagers stattfanden. Statt eines geschlossenen, transnationalen Eintretens für eine sozialistische Literatur unter realistischen Vorzeichen, das sich die Veranstalter von diesem Treffen versprochen hatten, wurde vielmehr die Forderung laut, moderne, avantgardistische, experimentelle Literatur auch in der DDR zuzulassen. Die ostdeutschen Schriftsteller:innen waren insbesondere bei den tschechischen, slowakischen und polnischen Kollegen als dogmatisch verschrien: „Zur Zeit ist dieser Druck aus dem sozialistischen Lager wirksamer als der des Gegners“, wie es kleinlaut in dem Bericht des DSV an die Ideologische Kommission des ZK der SED heißt. Viele Mitglieder des Verbands fürchteten daher „die Isolierung von ihren ausländischen Kollegen. “87

Wie in der Liga für Völkerfreundschaft, so nahm auch im Schriftstellerverband die Zusammenarbeit mit der Sowjetunion eine quantitativ und qualitativ herausragende Stellung ein. Selbstverständlich wird der ideologische Schulterschluss betont: „Wir [die Mitglieder des DSV] stützen uns in der internationalen Arbeit auf die vollständige Übereinstimmung und Einheit der Anschauungen der Leitung des sowjetischen Schriftstellerverbandes und unseres Verbandes." ${ }^{88}$ Die konkrete Ausgestaltung der Aktivitäten erfolgte unter „Anleitung“ durch das Ministerium für Auswärtige Angelegenheiten, das im Mai 1963 beispielsweise die Vorgabe macht, dass mit Blick auf die finanzielle Planung der Auslandsarbeit im DSV „,om gesamten Volumen

86 Vgl. Archiv des Schriftstellerverbandes der DDR, AdK, Signaturen SV 2831, 2837, 2838, $2839,2848$. Diese Kartei verzeichnet tatsächlich nur Einreisen in die DDR, die vom DSV veranlasst wurden, so dass bei weitem nicht alle literarischen Gäste aus dem Ausland aufgeführt sind. Konstantin Simonow zum Beispiel ist in dieser Kartei vielfach aufgeführt: 1953, 1961, 1966, 1967, 1971, 1976. Der Aufenthalt im Jahr 1964, bei dem er Grass und Johnson getroffen hat, ist allerdings nicht dabei, da er sich auf Einladung der Deutsch-Sowjetischen Freundschaftsgesellschaft in Ostberlin aufhielt.

87 Bericht des Sekretariats des Deutschen Schriftstellerverbandes an die Ideologische Kommission beim Politbüro des ZK der SED (Handschriftlich: „Entwurf 1963“). Archiv des Schriftstellerverbandes der DDR, AdK, Signatur SV 250. Vgl. Elke Scherstjanoi: Einleitung, in: dies. (Hrsg.) (Anm. 52), 7-47. 88 Koch (Anm. 59), 154. 
sozialistischer Länder 35-40 Prozent für die SU vorgesehen werden müssen“; auch müsse bei allen internationalen Veranstaltungen ,die Repräsentation der SU gesichert sein“ ${ }^{89}$ Nur zwei Monate später - um an diesem Beispiel auch Frequenz und Intensität der „Anleitung“ durch das MfAA zu demonstrieren - lädt die Kulturabteilung des MfAA den ersten Sekretär des Verbandes und den Leiter der Pressekommission des DSV zu einer „Aussprache“ ein zur „Verbesserung der Zusammenarbeit mit dem Deutschen Schriftstellerverband und zur Verstärkung der Information unserer Kulturattachés über Probleme der Literaturentwicklung in der DDR“. Von Seiten des MfAA wird ein qualitativer Fortschritt in der bilateralen Zusammenarbeit gefordert: „die Jahrespläne sollen künftig gemeinsame Symposien, Seminare, Aussprachen usw. enthalten“; weiterhin soll der Verband ,in der Auslandsarbeit eine gute Kaderpolitik betreiben, d.h. auf Grund politischer Überlegungen entscheiden, wer mit welchen konkreten Aufträgen ins Ausland entsandt wird und welche Schriftsteller mit den in die DDR einreisenden Gästen in Verbindung gebracht werden sollen.“ Angemahnt wird darüber hinaus „die exakte, regelmäßige Information unserer Kulturattachés“, „gründliche Auswertung der Auslandsarbeit“ und die Einschaltung der Botschaften schon „in der Vorbereitungszeit der Delegationen“, um „Einfluß auf die Festlegung des Aufenthaltsprogramms und die möglichst intensive auslandspropagandistische Ausnutzung der Delegation durch den Partnerverband“ zu nehmen. Im Hinblick auf die Inhalte, die an die Botschaften kommuniziert und im sozialistischen Ausland publik gemacht werden sollen, geht es ausschließlich um das Verhältnis der DDR-Literatur zur westdeutschen (unter Einschluss der Westberliner) Literatur. ${ }^{90}$ - Der Effekt dieser Zusammenkunft zeigt sich wiederum bereits einen Monat später: Bei einem Treffen des DSV mit dem Schriftstellerverband der UdSSR wird - den Vorgaben des MfAA folgend festgelegt, dass der „Inhalt der Delegationsreisen [...] ab sofort zu verändern“ sei und der „Delegationsaustausch auf neue Grundlagen gestellt“ werde. ${ }^{91}$

Das MfAA monierte im Jahr 1962 jedoch auch, dass bei der internationalen Arbeit auf dem Gebiet der Literatur sozialistische Länder zu stark gewichtet seien und nicht-sozialistische Länder mehr berücksichtigt werden müssten. ${ }^{92}$ In Bezug auf das „kapitalistische Ausland“ musste die Strategie des Schriftstellerverbands allerdings eine andere sein. Sie zielte im Wesentlichen darauf ab, über individuelle Kontakte zu linksgerichteten oder kommunistischen Schriftsteller:innen und Intellektuellen Verbindungen herzustellen und Einladungen zu realisieren, um das Bild der DDR positiv

89 Aktennotiz. Betr.: Anleitung im MfAA (SU) am 20. Mai 1963. Archiv des Schriftstellerverbandes der DDR, AdK, Signatur SV 877.

90 Archiv des Schriftstellerverbandes der DDR, AdK, Signatur SV 969, Bl. 28-32.

91 Aktennotiz, 20.08.1963. Archiv des Schriftstellerverbandes der DDR, AdK, Signatur SV 873/2, Bl. 67-68.

92 Vgl. die Anweisung des MfAA, der Austausch mit den sozialistischen Ländern müsse ,im Verhältnis zur Zusammenarbeit mit nichtsozialistischen Ländern stehen.“ Lediglich die Verbindungen zur Volksrepublik Polen seien weiterhin stärker auszubauen. Bundesarchiv (BArch), Signatur DR 1/20223. 
$\mathrm{zu}$ beeinflussen. So sollte, um ein Beispiel zu nennen, die Delegation, die zur Konferenz der COMES über Prosa 1962 in Leningrad entsandt wurde, „verpflichtet werden, für uns Verbindungen mit Schriftstellern des kapitalistischen Auslandes aufzunehmen

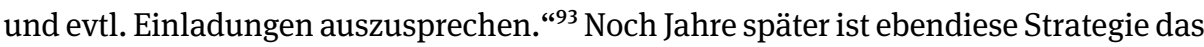
Mittel der Wahl im Kontakt zu nicht-sozialistischen Ländern:

Wie besonders auf dem Internationalen Schriftstellertreffen Weimar-Berlin [1965] zum Ausdruck kam, hatten eine Reihe von Verbandsmitgliedern sehr gute Verbindungen zu profilierten literarischen Persönlichkeiten in den entwickelten kapitalistischen Ländern. Die Auslandskommission und die Auslandsabteilung werden diese Verbindungen stärker als bisher in ihre Arbeit einbeziehen, um zu erreichen, daß solche Persönlichkeiten zu bestimmten Anlässen zum Besuch unserer Republik eingeladen werden. Diese Einladungen sollen dazu beitragen, neben den spezifisch literarischen Fragen das gesellschaftliche Leben unserer Republik im Ausland besser bekannt zu machen und gleichzeitig Voraussetzungen für das öffentliche Auftreten unserer Mitglieder zu schaffen..$^{94}$

Im Hinblick auf die „entwickelten kapitalistischen Länder“ sollen also vorhandene Kontakte genutzt und eher informelle Mittler eingesetzt werden, um ausländische Schriftsteller:innen mit möglichst hohem sozialen und symbolischen Kapital für die kulturpolitischen Zwecke der DDR heranzuziehen.

Insgesamt zeigt sich in den Berichten und Konzeptpapieren zur Auslandsarbeit, die der Schriftstellerverband in den 1960er Jahren auf den Weg brachte, eine gesteigerte Forderung nach Internationalisierung. Dabei werden die internationalen Aktivitäten immer wieder auch reaktiv begründet als Versuch, dem „ideologischen Unterwanderungsprozess der Bundesrepublik in den sozialistischen Ländern“ “5 ${ }^{95}$ wirkungsvoll entgegenzutreten, und „Taktik und Strategie des Gegners, die DDR kulturpolitisch zu isolieren, zurückzuweisen“. ${ }^{96}$ In einem 1963 verfassten Bericht des Sekretariats des Deutschen Schriftstellerverbandes an die Ideologische Kommission beim Politbüro des ZK der SED wird hervorgehoben, dass es in den vergangenen Monaten gelungen sei, die Auslandsbeziehungen des DSV erheblich zu erweitern; dennoch gebe es „in der Vorbereitung und Durchführung der Auslandsarbeit außerordentliche Mängel“:

In der Auslandsarbeit ist es notwendig, die Propagierung der nationalen und internationalen Rolle unserer sozialistischen deutschen Nationalliteratur und die objektive Information über die westdeutsche Literatur in den Mittelpunkt zu rücken. Dabei ist davon auszugehen, daß diese

93 Entwurf eines Arbeitsplans für das erste Halbjahr 1962. Archiv des Schriftstellerverbandes der DDR, AdK, Signatur SV 276.

94 Entwurf einer Konzeption der internationalen Arbeit des DSV. Archiv des Schriftstellerverbandes der DDR, AdK, Signatur SV 154, Bl. 57.

95 Entwurf einer Konzeption der internationalen Arbeit des Deutschen Schriftstellerverbandes. Archiv des Schriftstellerverbandes der DDR, AdK, Signatur SV 154, Bl. 56.

96 Bericht des Sekretariats an den Vorstand. Archiv des Schriftstellerverbandes der DDR, AdK, Signatur SV 152. 
Arbeit Bestandteil der einheitlichen Außenpolitik der DDR ist, daß sie der kulturpolitischen Zielsetzung des VI. Parteitages der SED entspricht, von der Übereinstimmung mit der Kulturpolitik der KPdSU aus.geht [sic!] und der Einheit des sozialistischen Lagers und der revolutionären Arbeiterbewegung in der ganzen Welt dient. ${ }^{97}$

Ebenfalls 1963 fand eine Mitgliederversammlung des DSV-Bezirksverbandes Berlin statt, die eigens dem Thema „Unsere Literatur in der internationalen Begegnung“ gewidmet war. Hans Koch, 1963 auf Betreiben der Kulturabteilung des ZK zum 1. Sekretär und stellvertretenden Vorsitzenden des DSV gewählt, berichtet darüber in der Zeitschrift des Schriftstellerverbandes NDL - Neue Deutsche Literatur - unter dem Titel Zu einigen Fragen unserer Auslandsarbeit:

Sie [die Auslandsarbeit des Schriftstellerverbandes] hat das Ziel, durch die Verstärkung der kulturellen Kontakte und Beziehungen die Wahrheit über die Deutsche Demokratische Republik zu verbreiten, der DDR und ihrer sozialistischen Literatur neue Freunde zu gewinnen und objektiv über die ideologisch-kulturellen Probleme, die sich aus dem Bestehen zweier deutscher Staaten ergeben, zu informieren. Sie ist darauf gerichtet, allen echten Leistungen der sozialistischen deutschen Nationalliteratur internationale Geltung zu verschaffen. ${ }^{98}$

Deutlich zeigt sich, dass die internationale Arbeit sich aus dem Impuls speist, die DDR-Literatur als eine spezifische, von der westdeutschen Literatur unterschiedene zu profilieren. Die Formel von den zwei deutschen Literaturen - eine verkommene westliche und eine sozialistisch-realistische, zukunftsträchtige - gehört zu den vehement vertretenden Basisannahmen des kulturpolitischen Programms der SED. Im Hinblick auf die internationale Durchsetzung dieser Position wird ein Maßnahmenkatalog entwickelt, der vor allem die verstärkte Präsenz der DDR-Literatur im Ausland bezweckt: Alle Aktivitäten sollten, so Hans Koch, dem Ziel einer „offensiveren und koordinierteren Propagierung unserer Literatur im Ausland“999 dienen.

\section{XI.}

Die internationale Kulturpolitik der DDR war darauf angelegt, die sozialistische Literatur in die Welt zu tragen; der Vektor war nach außen gerichtet. Selbstverständlich gab es aber auch Bestrebungen und Aktivitäten zur Vermittlung der ausländischen Literatur in die DDR; und gerade der Schriftstellerverband - als Vertretung der produktiven

97 Bericht des Sekretariats des Deutschen Schriftstellerverbandes an die Ideologische Kommission beim Politbüro des ZK der SED, 1963. Archiv des Schriftstellerverbandes der DDR, AdK, Signatur SV 250.

98 Koch (Anm. 59), 154.

99 Ebd., 160. 
Künstler - beklagte immer wieder Defizite in dieser Richtung. Auch wenn das Interesse im vorliegenden Aufsatz hauptsächlich den institutionalisierten Austauschbeziehungen, den Einladungsprogrammen und der Mobilität von Personen gilt und Bereiche wie Verlagswesen, Übersetzung, Literaturwissenschaft oder Publizistik, die für die Internationalisierung selbstverständlich ebenso relevant waren, kaum berücksichtigt werden, soll daher doch ein Seitenblick auf ein Thema geworfen werden, dem der Schriftstellerverband insistierende, wenngleich erfolglose Bemühungen widmete: der Plan, eine Zeitschrift für Weltliteratur - so der vorgeschlagene Titel - zu gründen.

Vorauszuschicken ist, dass in der Nachkriegszeit literarische Zeitschriften als Medium des internationalen Austauschs generell eine zunehmende Rolle spielten. Ein wichtiges Beispiel bietet die seit 1954 im Hanser Verlag erscheinende Zeitschrift Akzente, von Walter Höllerer Jahre vor seiner Berliner Zeit zusammen mit Hans Bender gegründet. Sie vermittelte dem deutschsprachigen Publikum die neue amerikanische Literatur ebenso wie den Nouveau Roman, die jugoslawische Literatur der Jahrhundertmitte sowie Texte von Autoren der Gruppe 47.100

In der DDR kam bereits in den 1950er Jahren die Idee einer Zeitschrift für internationale Literatur auf. ${ }^{101}$ In den Akten und Diskussionsberichten des Schriftstellerverbandes aus den 1960er Jahren kehrt insistierend die Forderung nach einem solchen literarischen Periodikum wieder. So wurde zum Beispiel 1960 im Vorstand des Schriftstellerverbandes der ausführliche Entwurf einer Zeitschrift für Weltliteratur vorgelegt. Vorbild der Zeitschrift war die 1955 in der SU gegründete Zeitschrift Inostrannaja Literatura - wörtlich übersetzt: Ausländische Literatur. Die zu gründende Zeitschrift sollte hauptsächlich Übersetzungen der Literatur sozialistischer Staaten wie auch der „fortschrittliche[n] Literatur der Länder mit anderen Gesellschaftsformen“ enthalten:

Den Hauptinhalt werden stets Übersetzungen von Prosa, Dramen, Lyrik usw. bilden. Die Auswahl muß vielfältig sein und schließlich einen guten Überblick verschaffen. Hinzu kommen Berichte über die literarische Situation in anderen Ländern, sowie kurze Übersichten über die Literaturgeschichte, besonders der jungen Nationalstaaten (Indonesien etc.). Diese Beiträge müßten im wesentlichen von unserer eigenen Literaturentwicklung ausgehen und nach Möglichkeit operativen Charakter haben.

100 Vgl. Michael Peter Hehl, Heribert Tommek (Hrsg.): Transnationale Akzente. Zur vermittelnden Funktion von Literatur- und Kulturzeitschriften im Europa des 20. Jahrhunderts, Berlin (im Druck). 101 Folgt man dem Beitrag des Schriftstellers Harald Hauser bei einer Diskussion der Mitgliederversammlung des Bezirksverbandes Berlin im DSV vom Dezember 1964, dann wurde bereits seit Mitte der 1950er Jahre das Projekt einer Zeitschrift mit dem Titel Weltliteratur diskutiert: „Da wir nicht alles drucken können, müssen wir uns überlegen, wie wir einen größeren Kreis von Menschen an diese Dinge heranführen können, wie wir sie literarisch informieren [...] und deshalb erhebe ich noch einmal - wie schon seit zehn Jahren - die Forderung nach einer Zeitschrift für Weltliteratur. [...] Warum nimmt der Verband keinen Einfluss?“ Stichwortprotokoll von den Diskussionsbeiträgen auf der Mitgliederversammlung des Bezirksverbandes Berlin am 17. Dezember 1964. Archiv des Schriftstellerverbandes der DDR, AdK, Signatur SV 339, Bl. 80 f., zit. nach Pamperrien (Anm. 69), 64. 
Die Zeitschrift „Weltliteratur“ wäre somit ein Mittel die provinzielle Enge in unserer Literatur zu überwinden, sie in Bezug zu stellen zu den Literaturen anderer Länder, besonders der sozialistischen, und aus ihren Erfahrungen zu lernen. Sie wird die primäre Bedeutung des sozialistischen Realismus hervorheben und gelegentlich nicht zaudern, kleine anprangernde Beispiele aus der Welt der formalen Experimente zu bringen. Rezensionen, Berichte, Informationen, Gedenkartikel werden zum ständigen Inhalt gehören. Der Zeitschrift kommt eine hohe politische Verantwortung $\mathrm{zu}$, es gibt keine Revue gleicher oder ähnlicher Art in deutscher Sprache. Sie muß immer interessant, unterhaltend, anregend (nicht zuletzt für unsere eigenen Schriftsteller) sein und dabei auch wissenschaftlichen Ansprüchen genügen. Für die freundschaftlichen Beziehungen der DDR zu vielen Staaten kann die Zeitschrift ein sehr wichtiges helfendes Werkzeug werden - einer der entscheidenden Gründe, das erste Heft so bald wie möglich erscheinen zu lassen. ${ }^{102}$

Am Ende werden Vorschläge für das Amt des Chefredakteurs gemacht: Anna Seghers hatte wohl ursprünglich - in einem Vorgängerentwurf - ihr Einverständnis erklärt; Otto Braun, kurze Zeit später Erster Sekretär des DSV, signalisierte seine Bereitschaft, während Bruno Kaiser, Bibliothekar des Instituts für Marxismus-Leninismus beim ZK der SED, bisher abgelehnt hatte. Man darf wohl davon ausgehen, dass, wäre dieser Entwurf realisiert worden, insbesondere die ,kleinen anprangernden Beispiele aus der Welt der formalen Experimente“ mit größtem Interesse wahrgenommen worden wären.

In den Folgejahren wiederholt sich diese Absichtserklärung mit zunehmender Dringlichkeit. Immer wieder, heißt es etwa in einem 1962 für eine Vorstandssitzung des DSV angefertigten Bericht, werde der Wunsch nach Gründung einer Zeitschrift Weltliteratur vorgetragen, es handele sich um eine seit Jahren schwelende Frage, aber man sei keinen Schritt weitergekommen. ${ }^{103}$ Ein Jahr später hat sich die Situation immer noch nicht geändert:

Es fehlt eine Zeitschrift für internationale Literatur. Die Behandlung und Veröffentlichung internationaler Literatur ist z.Zt. ausschließlich eine Sache des Verlages Volk und Welt und unserer Auslandsabteilung. Das Ergebnis dieser Arbeit ist völlig unzureichend, was besonders angesichts des bevorstehenden Welttreffens nicht länger geduldet werden kann. ${ }^{104}$

102 Archiv des Schriftstellerverbandes der DDR, AdK, Signatur SV 108.

103 Bericht für die Vorstandssitzung am 20. September 1962. Archiv des Schriftstellerverbandes der DDR, AdK, Signatur SV 129, Bl 34. In den Diskussionen, die im selben Jahr in der Akademie der Künste rund um die Absetzung von Peter Huchel als Chefredakteur der Akademie-Zeitschrift Sinn und Form geführt wurden, wurde zugunsten von Huchels eigenwilliger Komposition der Hefte vorgebracht, die Zeitschrift fülle mit ihrem derzeitigen Inhalt eine Lücke, sie ersetze eine Zeitschrift für Weltliteratur. Vgl. Inge Jens, Ulrich Dietzel (Hrsg.): Zwischen Diskussion und Disziplin. Dokumente zur Geschichte der Akademie der Künste (Ost) 1945/50-1993, Berlin 1997, 213.

104 Bericht des Sekretariats des Deutschen Schriftstellerverbandes an die Ideologische Kommission beim Politbüro des ZK der SED - 1963. Archiv des Schriftstellerverbandes der DDR, AdK, Signatur SV 250. 
Mit dem Hinweis auf Volk und Welt ist der Verlag angesprochen, der tatsächlich über Jahrzehnte in der DDR als literarisches ,Fenster zur Welt' fungieren sollte. ${ }^{105}$ Aus dem Schriftstellerverband kamen in der ersten Hälfte der 1960er Jahre allerdings immer wieder Versuche, eine weltoffenere Editionspolitik anzuregen. Erhellend ist in diesem Zusammenhang ein vermutlich im Dezember 1964 verfasstes Papier des DSV mit Bemerkungen „Zum Material des Ministeriums für Kultur über Probleme der gegenwärtigen Literatur- und Verlagspolitik“, in dem u. a. die Vorgaben des MfK bezüglich der Veröffentlichung moderner oder avantgardistischer Literatur kritisch kommentiert wurden:

Der Grundidee der Editionspolitik ausländischer Literatur, [...] stimmen wir vollinhaltlich zu. [...] Jedoch ist die Fragestellung in Bezug auf einige Literaturströmungen, die bisher in der DDR noch nicht vorgestellt sind [...] nicht richtig. Es geht nicht darum, bestimmte literarische Richtungen, wie die amerikanische Beat-Literatur, den nouveau roman in Frankreich oder das absurde Theater usw., weil sie modern oder im öffentlichen Gespräch sind, vorzustellen. Es kommt darauf an Lücken in der Publikation der bürgerlichen Weltliteratur der letzten Jahrzehnte zu schließen und damit nach und nach ein vollständiges, kritisch beleuchtetes Bild ihrer Entwicklung zu geben. Es kommt darauf an, die Bekanntschaft mit wichtigen und internationalen, wirksamen Werken der bürgerlichen Weltliteratur zu vermitteln und ihre Edition oder Nichtedition nicht von der Zugehörigkeit zu einer literarischen Strömung abhängig zu machen. ${ }^{106}$

Der Schriftstellerverband forderte eine systematische Prüfung der „bürgerlichen Weltliteratur“, um einen langfristigen Editionsplan zu erstellen. Auch wenn es einen derartigen übergreifenden Editionsplan nicht geben sollte, setzten sich doch auch in der DDR die Debatten um die Weltliteratur des 20. Jahrhunderts, um Proust, Joyce und Kafka, fort. In komplizierten, teils langwierigen und kleinteiligen Aushandlungsprozessen zwischen den beteiligten Institutionen und Akteuren - der Hauptverwaltung Verlage und Buchhandel im Ministerium für Kultur, den Verlagen, dem DSV - wurde es möglich, individuelle Autor:innen des modernistischen Kanons und der Gegenwartsliteratur aus dem nicht-sozialistischen Ausland zu publizieren. ${ }^{107}$

105 Vgl. Simone Barck, Siegfried Lokatis (Hrsg.): Fenster zur Welt. Eine Geschichte des DDR-Verlages Volk und Welt, Berlin 2003.

106 Archiv des Schriftstellerverbandes der DDR, AdK, Signatur SV 148/1.

107 Vgl. hierzu Peter Goßens, Monika Schmitz-Emans (Hrsg.): Weltliteratur in der DDR. Debatten Rezeption - Kulturpolitik, Berlin 2015; Siegfried Lokatis: Die Hauptverwaltung Verlage und Buchhandel, in: Simone Barck, Martina Langermann, Siegfried Lokatis: „Jedes Buch ein Abenteuer“. ZensurSystem und literarische Öffentlichkeiten in der DDR bis Ende der sechziger Jahre, Berlin 1997, 173-226; Carsten Gansel: Kanon und Kanonisierung in der DDR. In: Literarische Kanonbildung. Text + Kritik. Sonderband, hrsg. von Heinz-Ludwig Arnold, München 2002, 233-258. 


\title{
XII.
}

Kehren wir zurück zur Einladungspolitik und den internationalen Veranstaltungen in der DDR und betrachten einen Vorgang, der noch einmal auf andere Weise die Konkurrenz der Internationalisierung zwischen beiden Teilen der Stadt beleuchtet. Im Juli 1962 wendet sich Stefan Heym an Otto Braun, den Ersten Sekretär des Schriftstellerverbandes, um ihm eine Idee zu unterbreiten, die darauf hinausläuft, Höllerers Internationale Lesereihe, die im Winter 1961/62 Furore gemacht hatte, für Ostberlin zu adaptieren. Erkennbar zielt Heym auf die öffentliche, über die Grenzen des Landes hinausreichende Strahlkraft einer solchen Veranstaltung:

\begin{abstract}
Mein Vorschlag ist, daß die Mitglieder der COMES in der DDR diesen Herbst und Winter eine Serie großer literarischer Abende mit wichtigen ausländischen Schriftstellern durchführen, ungefähr nach dem Schema der im Vorjahr von Professor Höllerer in Westberlin veranstalteten Abende. Unsere Veranstaltungen sollten unter dem Patronat der internationalen COMES stattfinden und sich von denen Höllerers wohltuend dadurch unterscheiden, daß bei uns Ost und West vertreten sind. Ich denke dabei an Schriftsteller vom Range Graham Greenes, Moravias, Sartres, Twardowskys - um nur ein paar Namen zu nennen -, die auf diesen Veranstaltungen von den besten unserer Kollegen vorgestellt werden. ${ }^{108}$
\end{abstract}

Die Tendenz zur qualitativen Überhöhung und zum Superlativischen - große Abende, wichtige ausländische Schriftsteller, die besten Kollegen - zeigt den Anspruch, der hinter diesem Vorhaben steckt. Deutlich ist auch der Überbietungsgestus gegenüber dem Westberliner Vorbild: Heyms Veranstaltungen heben sich „wohltuend“ von der aufsehenerregenden Lesereihe Höllerers ab, indem sie Ost und West einbeziehen; sie sollen ebenso spektakulär, nur weniger einseitig sein. Zeigt sich darin einmal mehr die innerberlinische Ost-West-Konkurrenz im Hinblick auf literarische Internationalisierung, so kann man davon ausgehen, dass sich in Heyms Hinweis auf Höllerer auch eine strategische Überlegung verbirgt: Den offiziellen Kulturfunktionären soll darüber das symbolische Kapital verdeutlicht werden, das sich - analog zu Höllerer - mit einer solchen Aktivität erwirtschaften lässt. Das Patronat der COMES verstärkt wiederum diesen Effekt, indem es der geplanten Veranstaltungsreihe internationale Wahrnehmung und eine größere Reichweite sichert.

Von der Schirmherrschaft der Schriftstellervereinigung erhofft sich Heym aber noch mehr; oder anders gesagt, ohne Beteiligung der COMES hält er seinen Plan anscheinend nicht für realisierbar: Denn er schlägt vor, dass der COMES-Rat der DDR Kurt Stern, Paul Wiens und Heym selber - gemeinsam mit Otto Braun bei der sowjetischen Botschaft vorsprechen soll, um über den Kulturattaché die Veranstaltungsidee an die „sowjetischen COMES-Leute [...], deren Unterstützung wir für das Ganze

108 Brief von Stefan Heym an Otto Braun vom 26. August 1962. Bundesarchiv (SAPMO-BArch), Signatur DY 30, IV 2/9.06/273 
brauchen“ heranzutragen. Wenige Tage später wendet sich Otto Braun an Siegfried Wagner, den Leiter der Kulturabteilung beim Zentralkomitee der SED, um ihm gewissermaßen offiziell von Seiten des Schriftstellerverbandes den Vorschlag einer solchen internationalen Lesereihe zu unterbreiten. Braun betont, dass Anna Seghers, die Vorsitzende des DSV, Heyms Idee unterstütze, „wobei selbstverständlich die einzuladenden Schriftsteller sorgfältig auszuwählen wären“; und er vergisst nicht zu erwähnen, dass, „wenn solchen Veranstaltungen zugestimmt wird, auch entsprechende Mittel dafür bereitgestellt werden“ müssten. ${ }^{109}$ Obwohl vom ZK Zustimmung signalisiert wird und Otto Braun den Plan an Alfred Kurella weiterleitet ${ }^{110}$ - Kurella war allerdings der literaturpolitische und ideologische Scharfmacher im Politbüro des ZK der SED -, endet hier das Projekt einer Ostberliner Variante der Internationalen Lesereihe Höllerers. Die Initiative Heyms konnte sich offenbar nicht durchsetzen. Auch wenn über den weiteren Verlauf und die Gründe nichts bekannt ist, kann man festhalten, dass das primäre kulturpolitische Interesse von offizieller Seite nicht darin bestand, internationale Gegenwartsliteratur nach Ostberlin zu holen - auch und erst recht nicht einen Kommunisten wie Sartre, der „die Abrüstung der Kultur“ gefordert hatte -; vielmehr sollte ja vor allem die „sozialistische deutsche Nationalliteratur“ im Ausland beworben und die „Einheit des sozialistischen Lagers“ gefördert werden.

\section{XIII.}

Mit Stefan Heym verbindet sich auch ein kleiner Disput, der noch einmal in anderer Weise ein Licht wirft auf das Verhältnis von West- und Ostberliner Internationalität. In dem bereits erwähnten Gespräch zwischen Günter Grass, Walter Höllerer und Walter Hasenclever über Writers in Berlin wurde der Umstand, dass der DDR-Autor Stefan Heym seine Werke auf Englisch schrieb, als Opposition gegen die DDR ausgelegt. Hasenclever betonte:

Stefan Heym is a very special case. He went over to the East so that he could write in German again, after emigrating to America during the Nazi era. While he was abroad he wrote several quite successful books in English - The Crusaders, for example. When he arrived in East Berlin he continued to write in English because he refused to use the German language of the East zone. So his opposition takes the form of writing only in English; and then he has his books translated into German. ${ }^{111}$

109 Brief von Otto Braun, Deutscher Schriftstellerverband, an das Zentralkomitee der SED - Kulturabteilung, Genossen Siegried Wagner/Dieter Heinze vom 31. Juli 1962. Bundesarchiv (SAPMO-BArch), Signatur DY 30, IV 2/9.06/273

110 Vgl. Walenski (Anm. 65), 38.

111 Höllerer, Grass, Hasenclever (Anm. 23), 112. 
Heym verwahrte sich in einem - englisch geschriebenen - Brief entschieden gegen die Unterstellung, die Wahl der Sprache in seinen Werken habe etwas mit Dissidententum zu tun und wies darauf hin, dass er sowohl auf Englisch als auch auf Deutsch publiziere. Die USA habe er nicht aufgrund eines diffusen Wunsches, wieder deutsch zu schreiben, verlassen, sondern - wie Thomas Mann, Bert Brecht und Charlie Chaplin auch - aufgrund des McCarthyismus, also der antikommunistischen Hetzjagd zu Beginn der 1950er Jahre. ${ }^{112}$ Bezeichnend an diesem Schlagabtausch sind die wechselseitigen Zuschreibungen: Diese Form der Internationalität konnte es aus Westperspektive im Osten offenbar nicht geben; wenn ein Ostberliner Autor auf Englisch schreibt, dann erscheint das aus der Sicht der Westautoren quasi notwendig als subversives Verhalten. Ohnehin stellt sich im Verlauf des Gesprächs die Frage, wer denn als Berliner Autoren - Writers in Berlin - anzusprechen sei; und Höllerer und Grass geben zu verstehen, dass es, bis auf wenige - Huchel und Bobrowski werden genannt -, für sie in Ostberlin kaum erstzunehmende Schriftsteller oder Schriftstellerinnen gibt.

Den Umstand, dass die drei ohne Ostberliner Beteiligung über Berliner Autoren reden, begründen sie implizit durch ihren Hinweis auf die Schwierigkeiten des Kontakts über die Mauer hinweg. Heym hingegen, der in der Tat zeit seines Lebens ein kritisch gespanntes Verhältnis zur DDR hatte und später etliche seiner Werke nur in der BRD publizieren konnte, fühlte sich durch die etwas gönnerhaften Ausführungen ${ }^{113}$ der drei Westberliner über Ostberliner Autoren veranlasst, die Reiseschwierigkeiten

112 Brief von Stefan Heym an den Herausgeber des Atlantic Magazine vom 22. Dezember 1963. Nachlass Walter Höllerer, Literaturarchiv Sulzbach-Rosenberg, Signatur 03WH/AA/7,15.

113 „HÖLLERER: I'd like to make a few remarks now about the East Berlin writers. The journal Sinn und Form [Meaning and Form] still exists there; it was edited until recently by Peter Huchel, but they've now got rid of him. Then there are writers like Bobrowski, who won the prize of the Group 47. We can call such literary men 'Berlin writers,' though their inclusion has become more difficult since the Wall.

GRASS: Yes, but there are also a large number of writers - serious writers, too - with whom it was impossible to discuss literary questions even before the building of the Wall, and now, of course, it's out of the question.

HÖLLERER: lt's very difficult to make any generalizations about relations between writers in East and West Berlin - each individual is different. Some are genuine writers, others are Party officials. Depending on which they are, you can discuss things with them or not.

GRASS: Writers with whom you could discuss literary subjects are the very ones that you must now try to leave alone - otherwise you might get them into serious trouble; the others are to be avoided at all costs.

HASENCLEVER: Can you still see Bobrowski or the poet Christa Reinig, for example?

GRASS: They don't come to West Berlin, and we can't go over there. Christa Reinig never comes. Bobrowski finds it very difficult to get a pass.

HÖLLERER: He was here for half a day when he received the prize of the Group 47. He got a visa for that, but it was a great exception. We who have West Berlin passes can’t go over to East Berlin at all.“ Höllerer, Grass, Hasenclever (Anm. 23), 111. 
innerhalb Berlins herunterzuspielen; er schlug eine gemeinsame Diskussion auf Westberliner Boden vor und verwettete seine nächsten westdeutschen Tantiemen darauf, die Reiseerlaubnis zu bekommen. Damit sollte er recht und zugleich nicht recht behalten: Während er beispielsweise am 5. November 1965 in Siegmunds Hof, der Studentenstadt in Westberlin, lesen konnte, scheiterte 1966 ein von denselben Veranstaltern geplantes Gespräch zwischen Heym und Grass tatsächlich am Ausreiseverbot für Stefan Heym. ${ }^{114}$

Zwischen diesen beiden Veranstaltungen, im Dezember 1965, fand allerdings das 11. Plenum des Zentralkomitees der SED statt, das als sogenanntes ,Kahlschlagplenum“ in die Geschichte eingegangen ist und das Ende einer liberaleren Ära der früheren 1960er Jahre markiert. Im Mittelpunkt der Anklage durch Honecker und das Politbüro standen Autoren wie Stefan Heym, Heiner Müller, Wolf Biermann, Werner Bräunig u. a., aber auch das Versagen der Kontrollgremien wurde angeprangert. Bücher wurden in der Folge verboten, Stücke, Filme, ein ganzer Jahrgang der DEFA verschwand in den Archiven. ${ }^{115}$ Auch für den Schriftstellerverband und nicht zuletzt für dessen Auslandsabteilung hatte das Plenum, im Verein mit der Auswertung und kritischen Beurteilung des Internationalen Schriftstellertreffens von 1965, Folgen: Der Vorstand versammelte sich zur Selbstkritik, ein Teil des Vorstands trat zurück, der Sekretär der Auslandsabteilung wurde durch einen bisherigen Mitarbeiter des Ministeriums für Auswärtige Angelegenheiten ersetzt, Wolfgang Joho, Chefredakteur der Zeitschrift des Schriftstellerverbandes $N D L$, musste seinen Platz räumen.

\section{XIV.}

Literatur in Berlin nach dem Mauerbau war in der Gefahr, randständig zu werden; sie war auf beiden Seiten und aus unterschiedlichen Perspektiven dem Verdacht der Provinzialität ausgesetzt. Und sie war politisch, politischer als anderswo: nicht bzw. nicht notwendig von den Inhalten her, sondern von dem Ausmaß an kulturpolitischer Instrumentalisierung und den gewünschten oder erwarteten Effekten her. In Ostberlin wurde die Kultur ohnehin als wichtiges Instrument internationaler Durchsetzung und staatlicher Selbstbehauptung angesehen; aber auch in Westberlin wurde, mehr als je zuvor und mehr als an jedem anderen Ort der Bundesrepublik, die politische Bedeutung der Kultur veranschlagt und in diesem Sinn die Internationalisierung vorangetrieben. Dabei sind die dominanten Zielrichtungen gegenläufig: Während es in

114 Vgl. Helmut Peitsch: Remigration, Übersiedelung, Westarbeit, in: Ulrich von Bülow, Sabine Wolf (Hrsg.): DDR-Literatur. Eine Archivexpedition, Berlin 2014, 22-35, hier: 32 ff.; vgl. Roland Berbig: Allzeit Ostberlin im Auge (Günter Grass), in: ders. (Hrsg.): Stille Post (Anm. 5), 218-237, hier 220. 115 Vgl. Günter Agde (Hrsg.): Kahlschlag. Das 11. Plenum des ZK der SED 1965. Studien und Dokumente, 2., erw. Aufl., Berlin 2000. 
Westberlin vor allem darum ging, die internationale Moderne in die Stadt zu holen und dadurch Teilhabe, Weltläufigkeit und Westbindung zu demonstrieren, zielte die Ostberliner Literaturpolitik vorrangig auf Außenwirkung und internationale Vermittlung der ,sozialistischen deutschen Nationalliteratur‘.

Diese Phase der Kulturentwicklung Berlins fällt bekanntlich in die Amtszeit von Willy Brandt als Regierendem Bürgermeister. Brandt pflegte den Kontakt gerade zur Literatur, er hielt mehrfach Treffen mit Schriftstellern ab; und die Bekanntschaft mit Günter Grass (der 1965 für ihn Wahlkampf machte) ${ }^{116}$ und mit Walter Höllerer geht auf diese Zeit zurück. 1966, mit der Großen Koalition, wechselte Brandt in die Bundespolitik und wurde Außenminister. Kurz nach Amtsantritt hielt er eine programmatische Rede, die als Zäsur in der Geschichte der Außenkulturpolitik der BRD gilt:

Die Auswärtige Kulturpolitik ist zu einem der drei Pfeiler moderner Außenpolitik geworden. Sie steht gleichwertig neben der Diplomatie im engeren Sinne und der Außenhandelspolitik. [...] Neben die nationale Repräsentation ist die internationale Kooperation getreten. Es ist in unserer Zeit ein Gebot der politischen Verantwortung, den Austausch zwischen Wissenschaftlern, Schriftstellern und Künstlern aller Länder zu fördern. Dieser Austausch nutzt allen, die sich an ihm beteiligen. Denn auf den Gebieten der Kultur gibt es im Grunde nur Entwicklungsländer. ${ }^{117}$

Auch wenn sich Brandts Statement nicht einfach auf seine Erfahrungen in Berlin zurückführen lässt: In jedem Fall kann man festhalten, dass die Berliner Situation der frühen 1960er Jahre eine weitreichende Schule war, in der eine solche Aufwertung der Außenkulturpolitik bereits praktisch realisiert und vorgeführt wurde. Das Ausmaß einer gezielten Internationalisierung - in Westberlin und auf andere Weise auch in Ostberlin - war neu; geboren aus der Konkurrenz der Systeme und befeuert durch die spezifische Situation der Stadt diesseits und jenseits der Mauer, war sie doch wegweisend für die Zukunft des Literaturbetriebs.

116 Vgl. Willy Brandt, Günter Grass: Der Briefwechsel, hrsg. von Martin Kölbel, Göttingen 2013.

117 Willy Brandt: Bedeutung und Aufgaben der Auswärtigen Kulturpolitik (Ausführungen von Außenminister Brandt anlässlich der Veröffentlichung des Tätigkeitsberichtes 1966 der Kulturabteilung des Auswärtigen Amtes), Bulletin der Bundesregierung Nr. 71 (05.07.1967), 613-614, hier: 613. 
